$$
\begin{aligned}
& \text { مدى المتوافر من أبعاد الإبداع الاستراتيجي المني }
\end{aligned}
$$

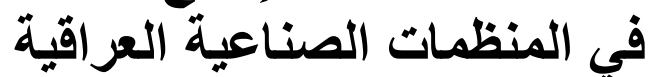

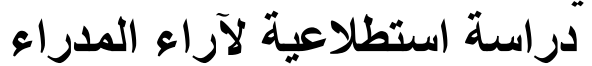

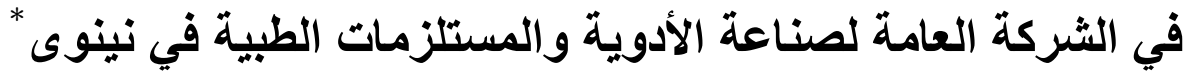

$$
\text { أيمن جاسم محمد }
$$

كلية الإدارة والاقتصاد -جامعة الموصل

Ayman_a77n@yahoo.com

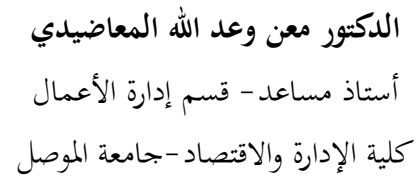

الدكتور معن وعد الله المعاضيدي

أستاذ مساعد - قسم إدارة الأعمال

كلية الإدارة والاقتصاد -جامعة الموصل

maanalamaadhedee@yahoo.com

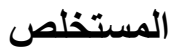

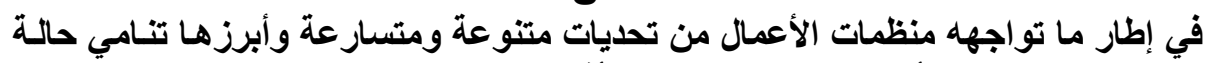

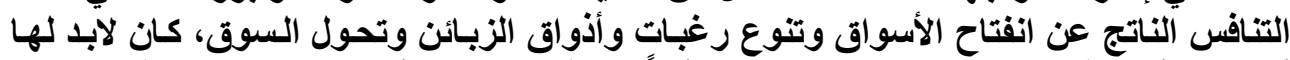

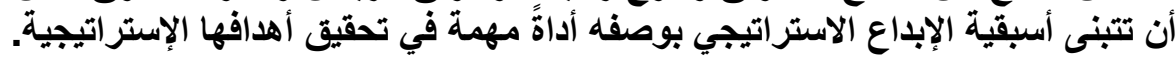

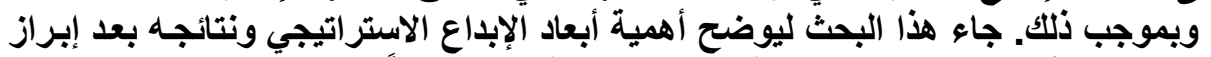

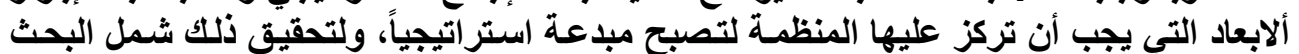

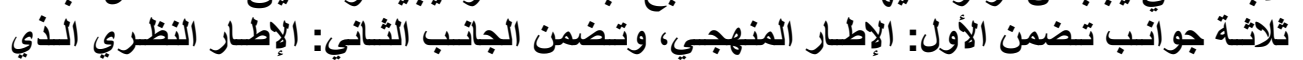

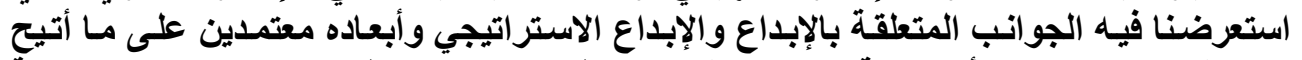

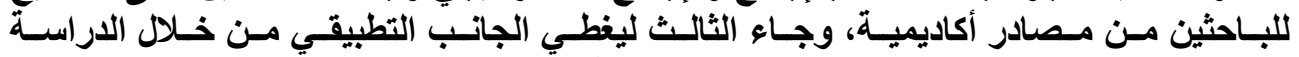

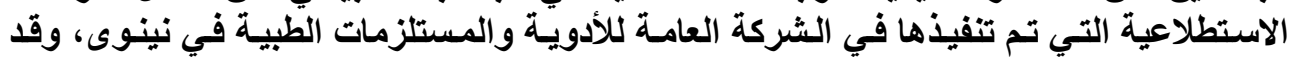

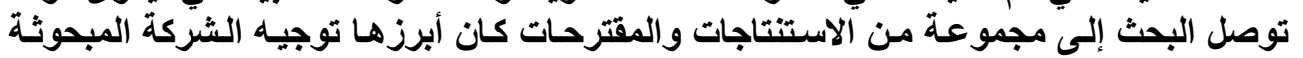

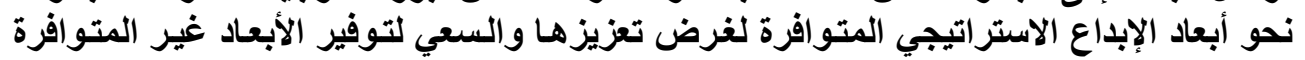
للإبداع الاستر اتيجي فيها.

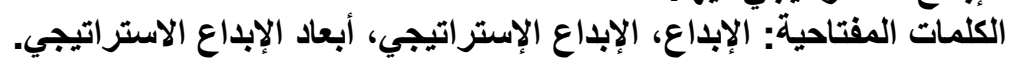

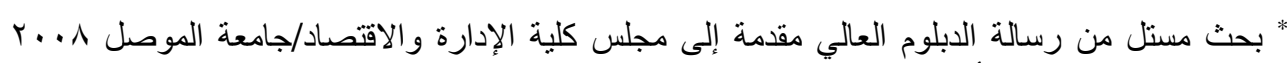

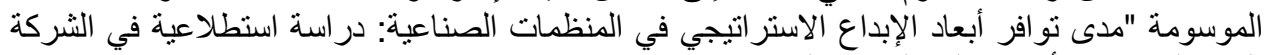

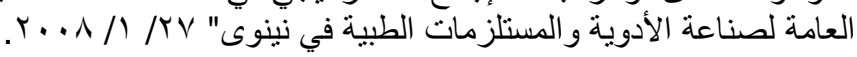

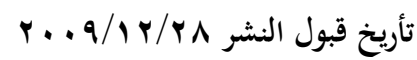

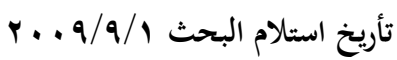




\title{
Extent of Availability of Strategic Innovation Dimensions in Iraq Organizations of Industrial A Pilot Study at Managers Opinions in Nineveh State Company for Drugs Industry and Medical Appliances
}

\author{
Maan W. Al-Maadhedee (PhD) \\ Assistant Professor \\ Department of Business Administration \\ University of Mosul
}

\author{
Ayman J. Mohammed \\ Researcher \\ Department of Business Administration \\ University of Mosul
}

\begin{abstract}
Through the fierce and accelerated challenges faced by business organizations, the rise of competition brought about by opening of markets and the diversity of the wishes and desires of customers and a market shift, it used to face these conditions via adopting the primacy of strategic innovation as an important tool for achieving strategy. The research demonstrated the importance and the dimensions of strategic innovation and results after the focus on the dimensions of strategic innovation that should be confirmed in organization to have innovative strategy. To achieve the goal of the research, there were three aspects; the first is methodological framework. To ensure the second aspect, theoretical aspects reviewed the innovation strategy, innovation and dimensions. The study depended on the available academic resources among researchers. The third covered the practical aspects through the survey implemented in the State Company of Drugs Industry and Medical Appliances in Nineveh. The research found a set of conclusions and proposals, the most notable is directing the company examined to the dimensions of innovation strategy available for the purpose of promotion and work to provide dimensions not available to the innovation strategy.
\end{abstract}

Key Words: Innovation, Strategic Innovation, Strategic Innovation Dimensions.

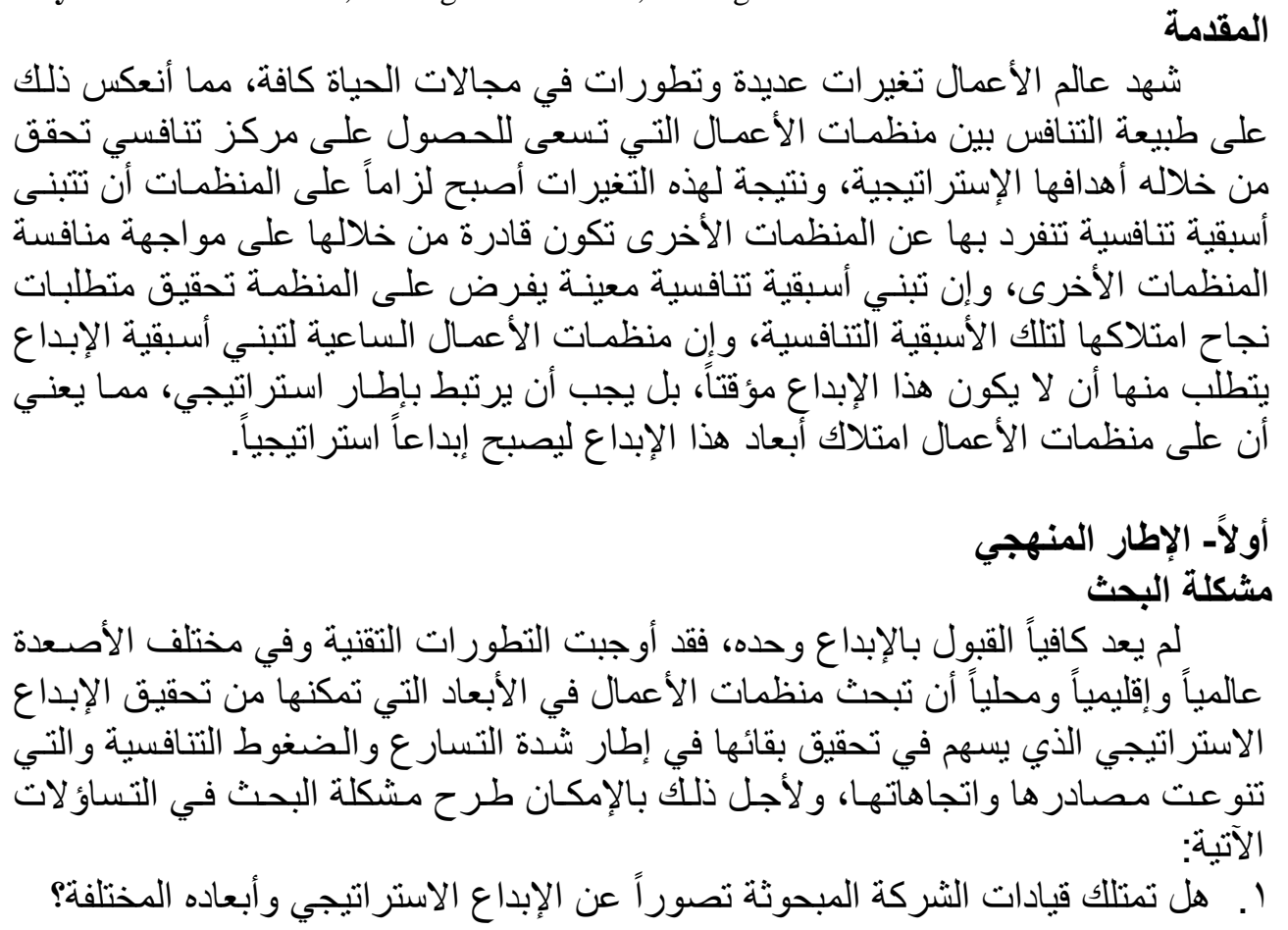




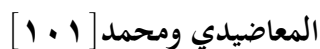

Y. . ما مدى المتو افر من أبعاد الإبداع الاستر اتيجي في الثركة المبحوثة؟ الإبه

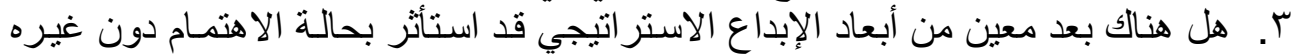

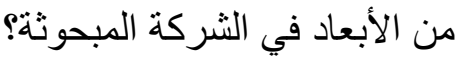
ء. . ما المطلوب من الثعاد في الثركة المبحوثة القيام به لتعزيز أبعاد الإبداع الاستر اتيجي المتو افرة

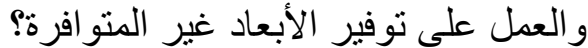

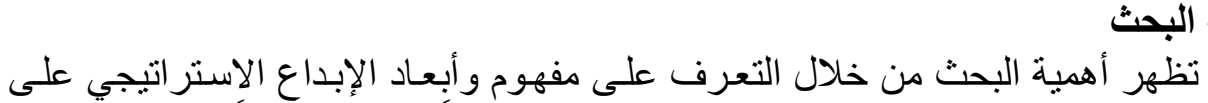

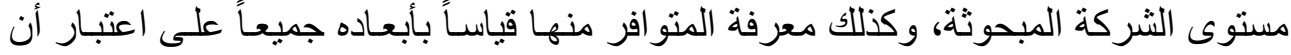

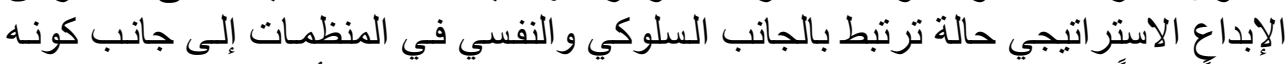

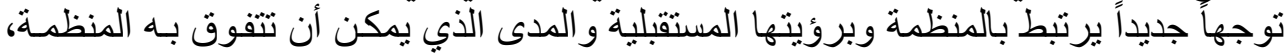

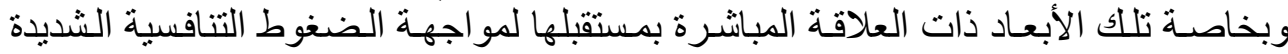
و المتسار عة، و المحصلة تحقيق أهدافها بالبقاء و الاستمرار .

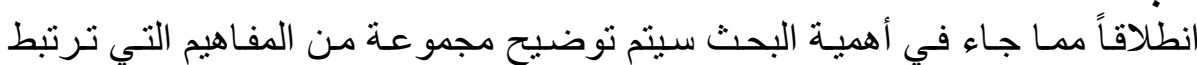
أ هداف البحث

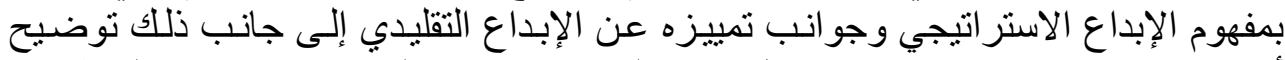

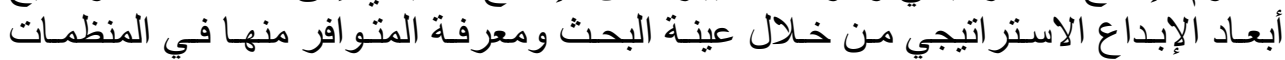
الصناعية العر اقية، عليه بالإمكان تحديد أهداف البحث البحث بالفقر ات الات الآتية:

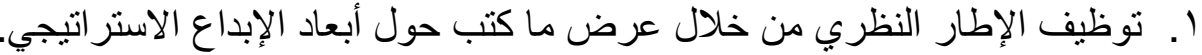

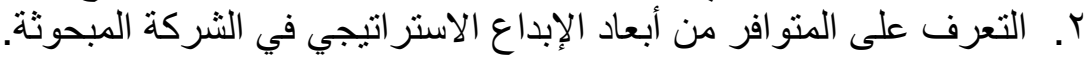

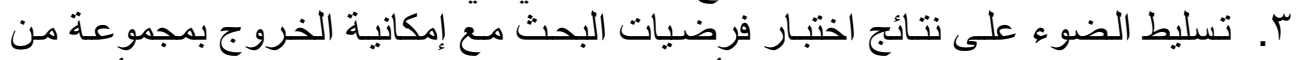
الاستتناجات و المقترحات التي من شأنها تعزيز الاهنمام بالإبداع الاستر اتيجي وأبعاده.

فرضية البحث

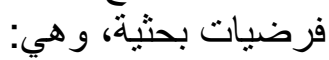
ا ا ـ تتو افر أبعاد الإبداع الاستراتيجي في الثركة المبحوثة.

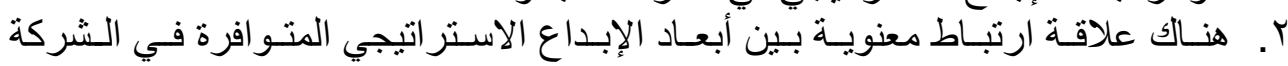

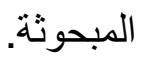

لغرض الإجابة على التخمينات الواردة في فرضية البحث، ووصدو لاً إلى أهدافه فقد منهج البحث

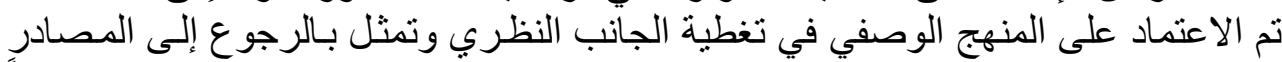

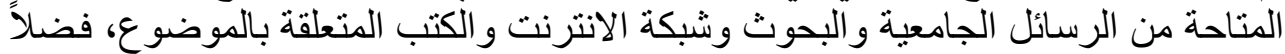

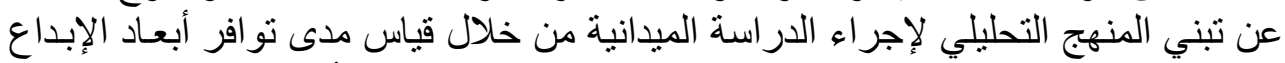
الاستر اتيجي في الثركة المبحوثة وقياس علاقة الإرتباط بين هذه الأبعاد. 


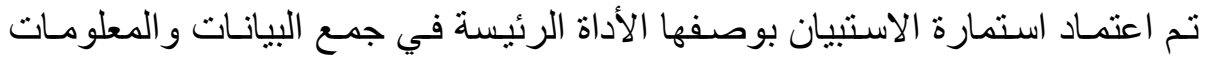

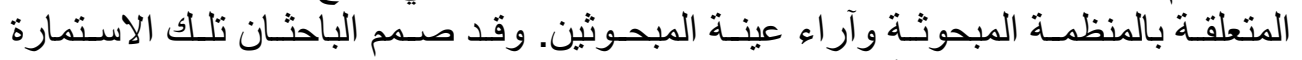

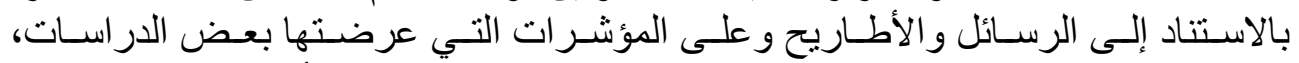

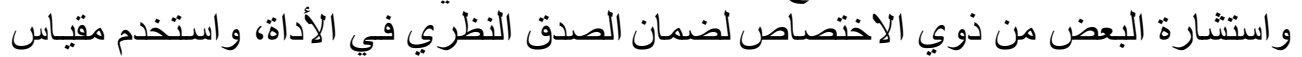

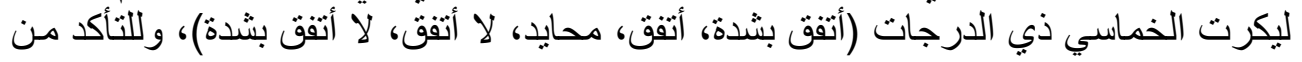

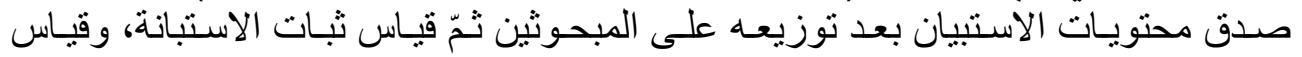

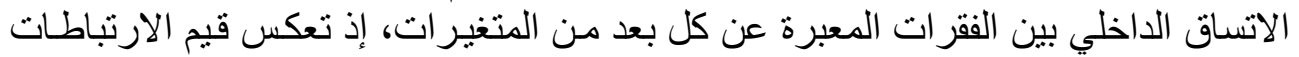

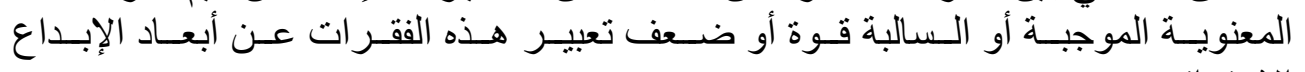
الاستر اتيجي. أ أ ـ قياس ثُبات الاستبانة لغرض التعرف على على مدى صلاحية المقياس وملاعمته تم استخدام مقياس (كرومباخ

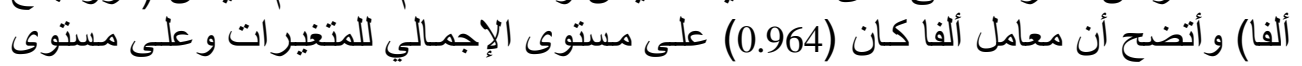

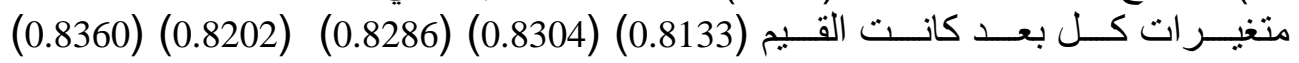

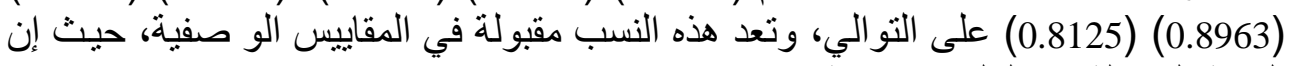

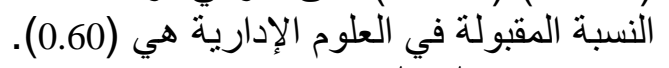

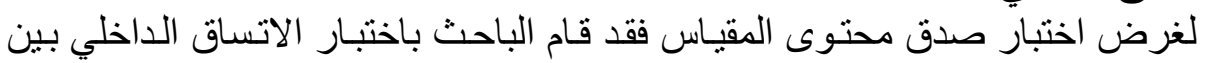

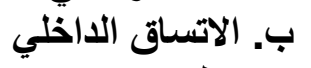

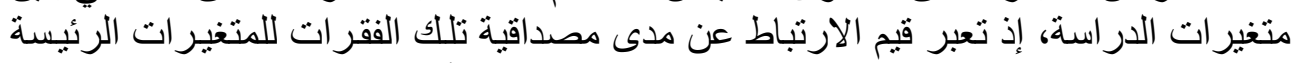

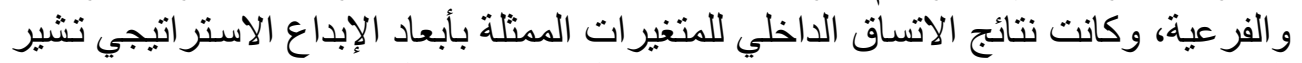

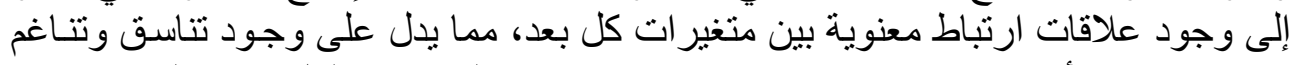
بين متغير ات الأبعاد وهذا بدوره يؤشر إمكانية مئن فياس هذه المتغير ات لكل بعد تمثله.

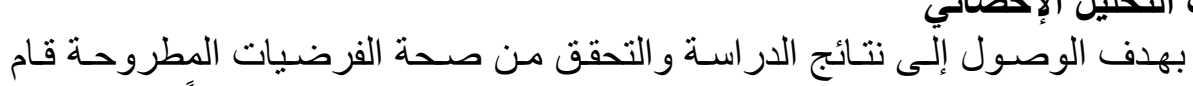

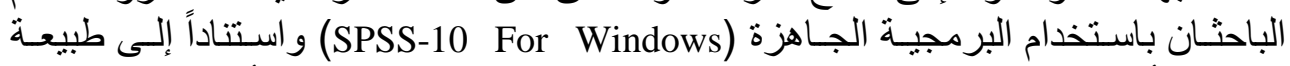
توجهات أهداف الدراسة والبان وضامين فرضياتها فقد استعانا بمجموعة من الأدوات الإحصائية

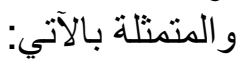

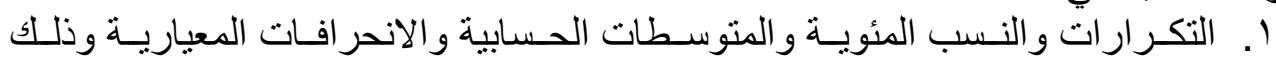

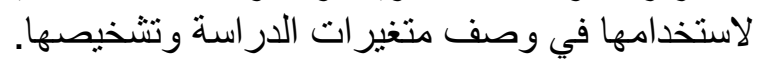

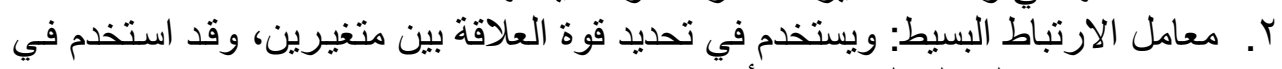

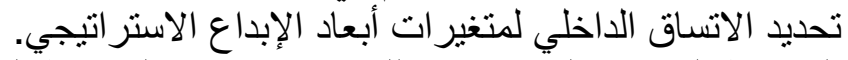

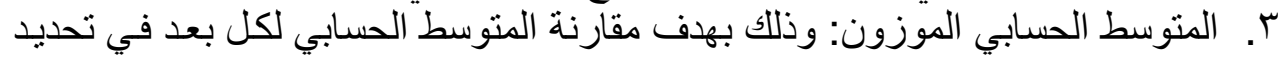

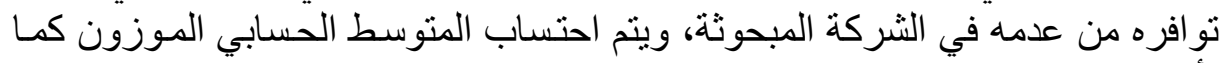

$$
\bar{y}=\frac{\sum w_{i} y_{i}}{\sum w_{i}} \text { * }
$$
يأني: تون " الراوي، خاشع محمود، (919 (19) مدخل إلى الإحصاء، مديرية دار الكتب للطباعة والنشر، جامعة 


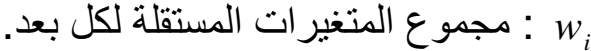

$$
\begin{aligned}
& \text { المنوسط الحسابي لكل بعد. } \\
& \text { S } \sum w_{i}
\end{aligned}
$$

بالإمكان تقسيم حدود البحث إلى ما يأتي:

حدود البحث

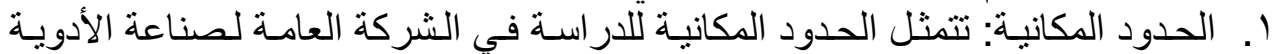

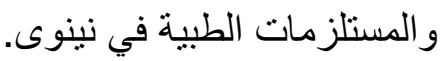

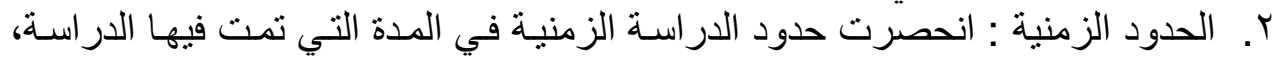

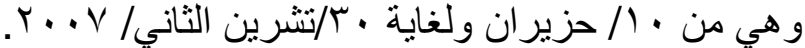

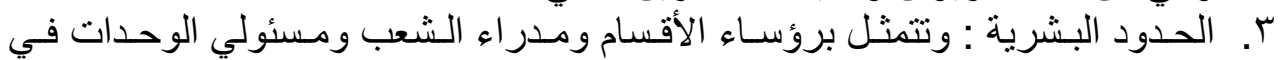

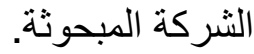

\section{صعوبات البحث} ا. . شحة المصادر العربية وحتى الأجنبية التي تناولت مفهوم الإبداع الاستر اتيجي.

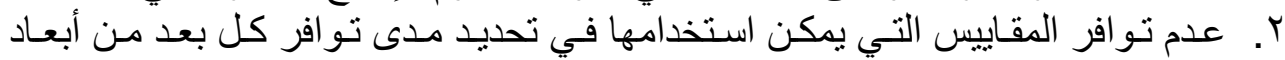

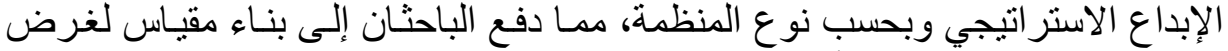
استقصاء مدى توافر تللك الأبعاد في المنظمة المبحوثة.

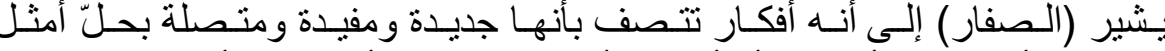

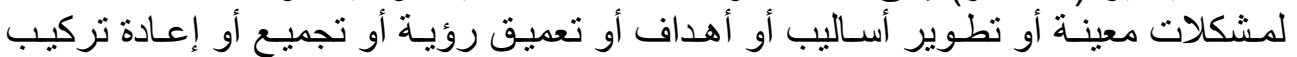

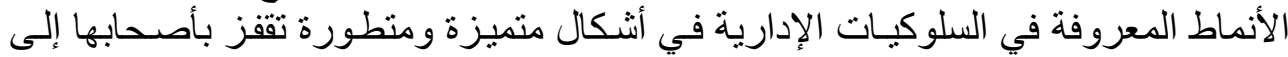

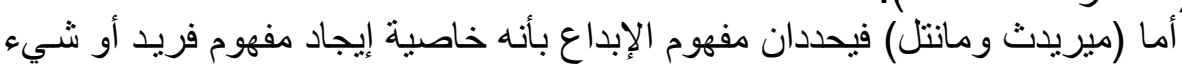
ثُانياً الإطار النظري مفهوم الإبداع الاستر اتيجي الميري

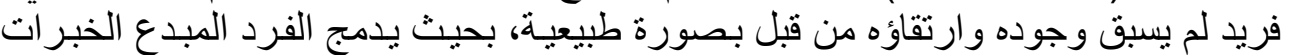

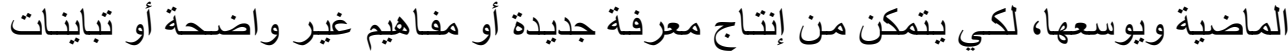

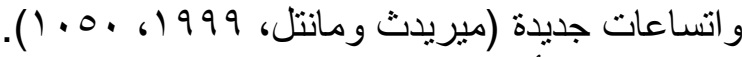

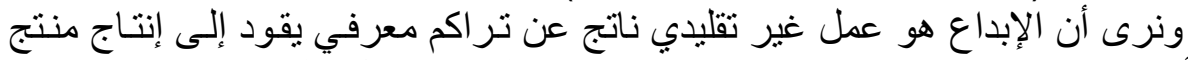

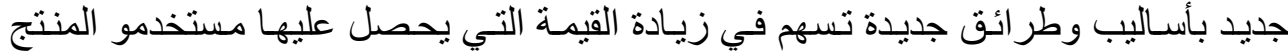
ويعزز من الميزة التنافسية للمنظمة.

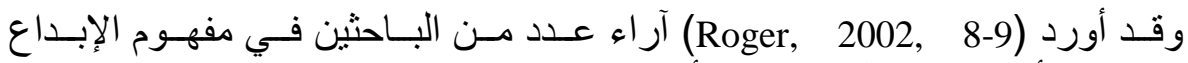

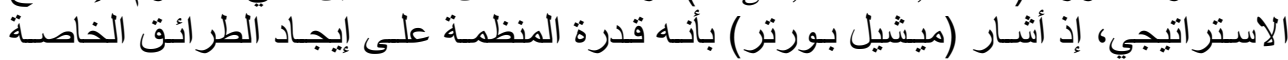

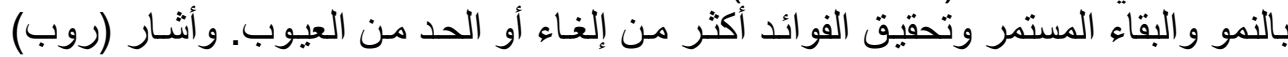

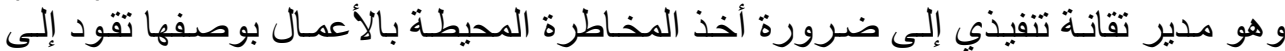

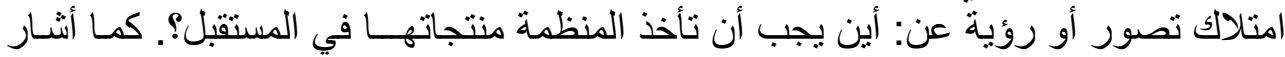

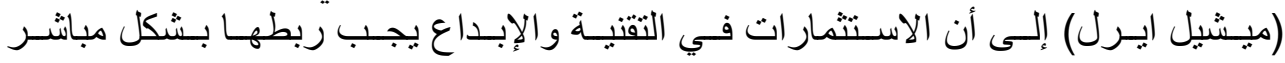


بإستر اتيجية المنظمـة، في إثـارة منـه إلى مـا يتطلبـه الإبـداع الاستر اتيجي من استثمار ات مادية وبشرية.

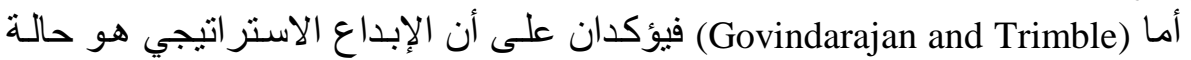

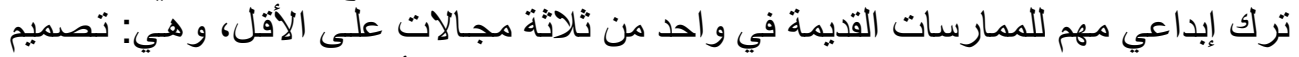

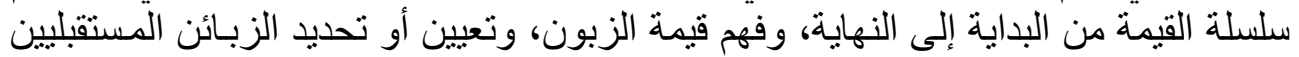

(المحتملين) (Govindarajan and Trimble, 2005, 69)

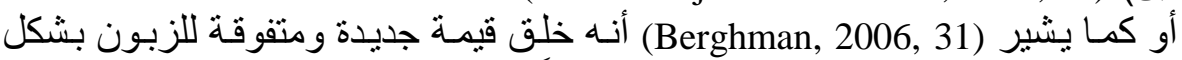

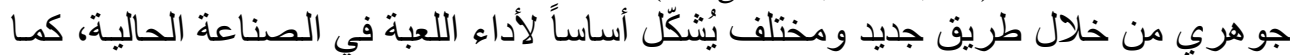

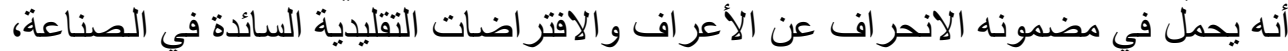

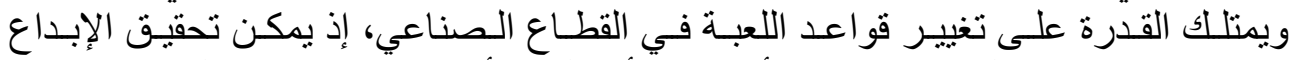

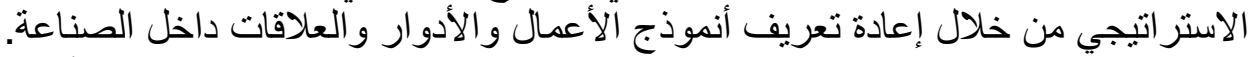

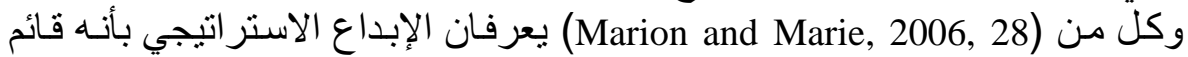

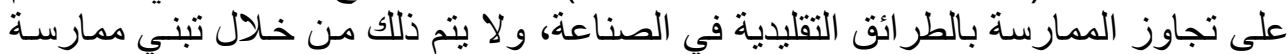

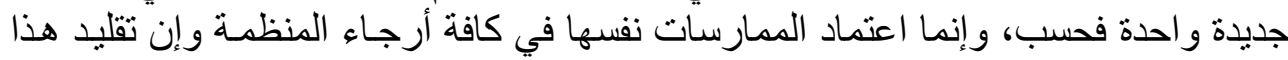
النوع من الإبداع يتطلب محاكاة نظام معقد في إطار حالّة الاعتماد الداخلي بين مكونات النات هذا

أمـا (Sniukas, 2007, 24) فإنـه يحدد مفهوم الإبداع الاستر اتيجي بأنه: إطـار عمل فئل

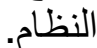

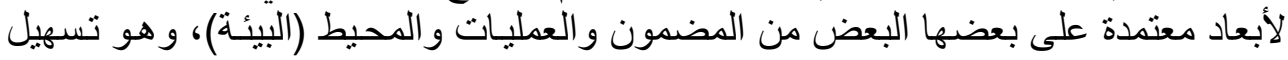

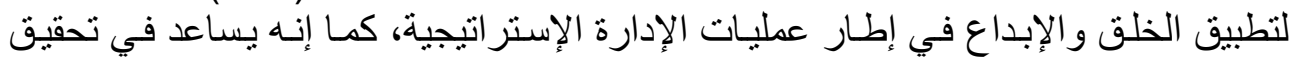

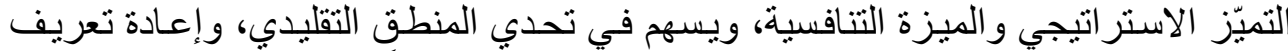

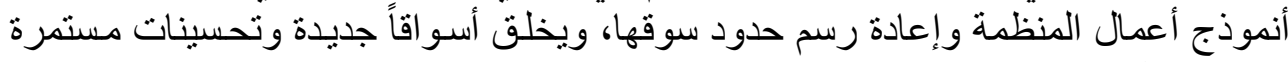
في قيمة كل من الزبائن و المنظمة.

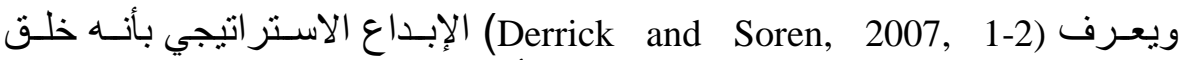

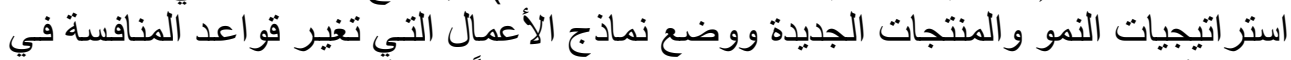

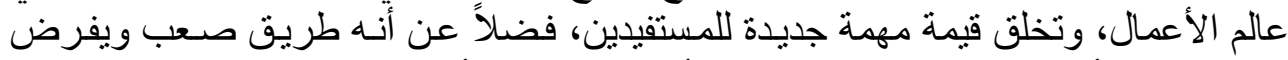

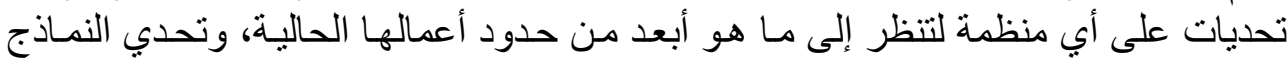

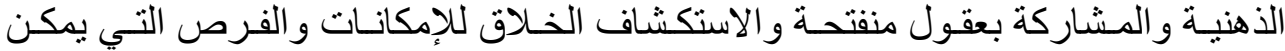

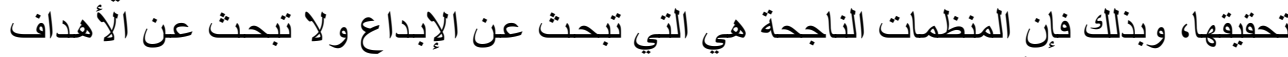

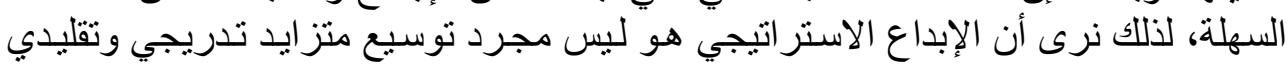

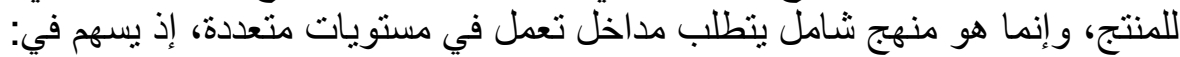

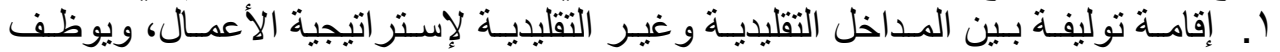

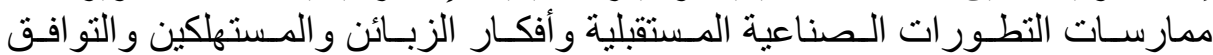

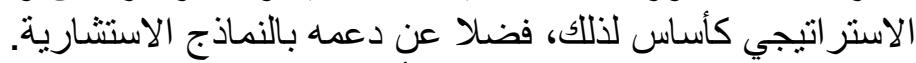

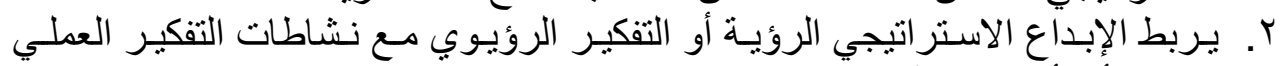

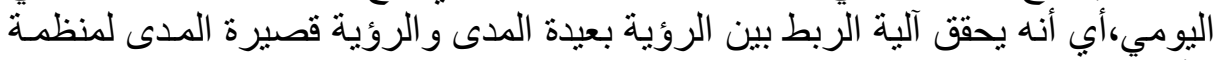

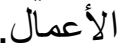

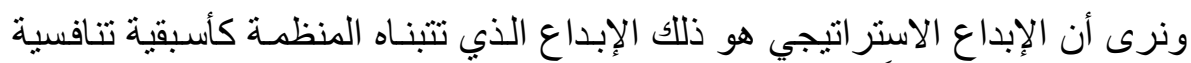

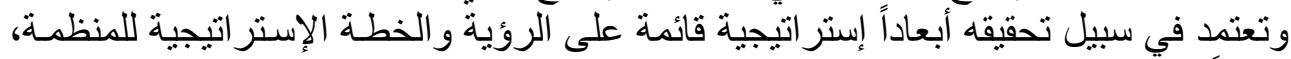

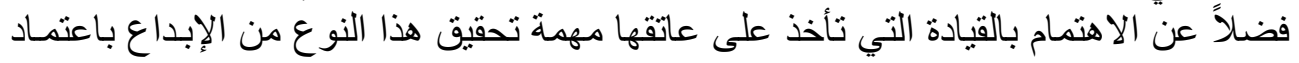




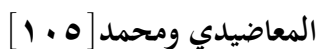

فرق متخصصة تجعل منهـ إبداعاً متكرراً ومستمر اً ومقصوداً، ويقود إلى تحقيق أهداف

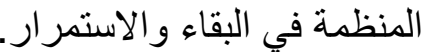

الحاجة إلى الإبداع الاستراتيجي

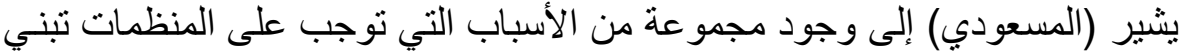

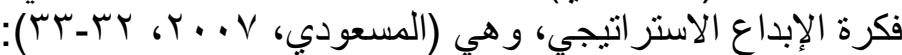

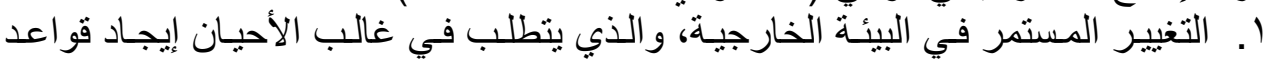

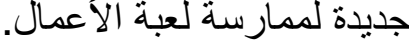

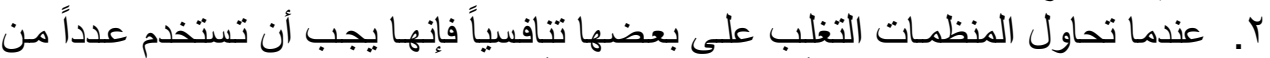

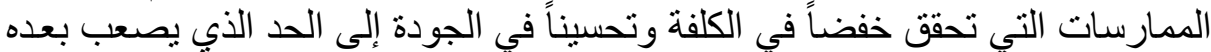

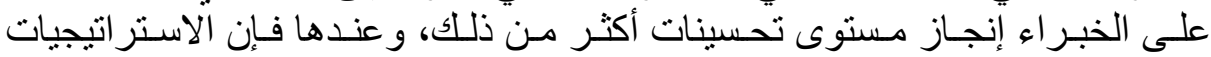
الجديدة للأعمال يجب أن أن توضع موضع التنفيذ.

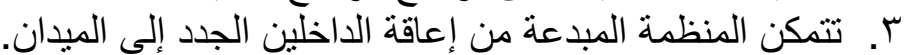

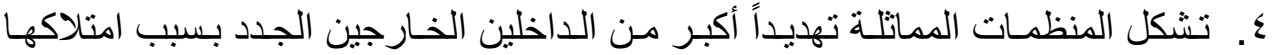

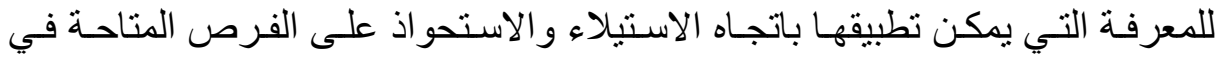

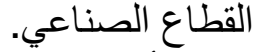

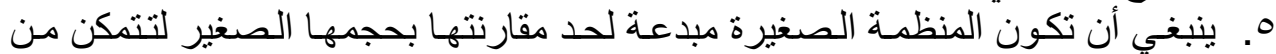
البقاء و التعايش مع المنظمات الكبيرة في الميدان.

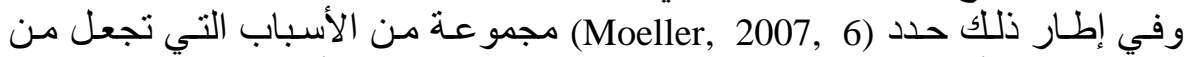
الإبداع الاستراتيجي أولوية للى الكثير من المنظمات في السنوات الألخئ الأخيرة وهي:

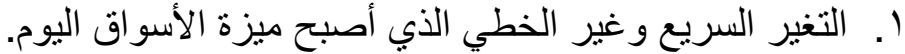

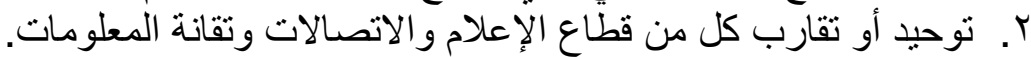

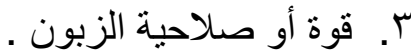
ع. التقنيات الرقمية الحديثة التي حولت قطاع الخدمات إلى أنموذج جديد.

جوانب التمييز بين الإبداع والإبداع الاستراتيجي التيراتي

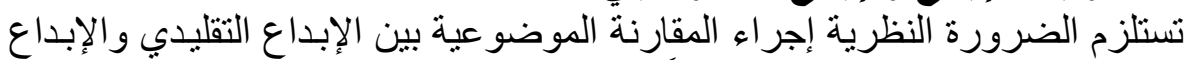

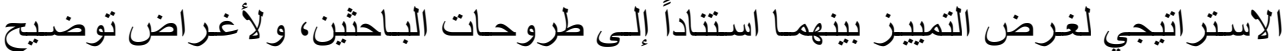

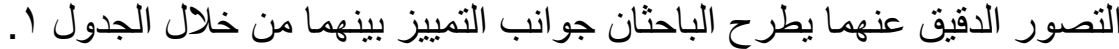

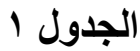

مقارنة بين الإبداع التقليدي والإبداع الاستراتيجي

\begin{tabular}{|c|c|}
\hline الإبداع الاستر اتيجي & الإبداع التقليدي \\
\hline 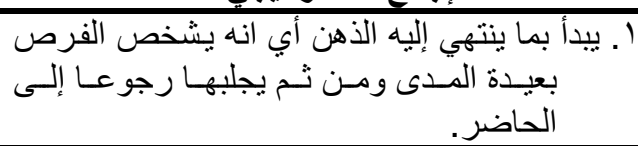 & 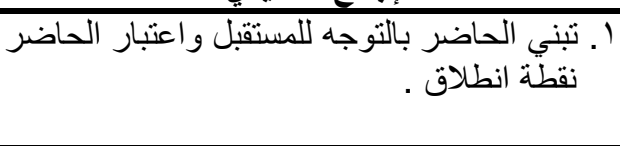 \\
\hline ب. بيقترضع التعامل ميع الذهنية التي تحطم القواعد & كاع وليه \\
\hline
\end{tabular}




\begin{tabular}{|c|c|}
\hline الإبداع الاستر اتيجي & الإبداع التقليدي \\
\hline ن خلق ميادين تتافسية جديدة للأعمال. & 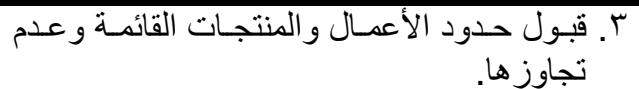 \\
\hline 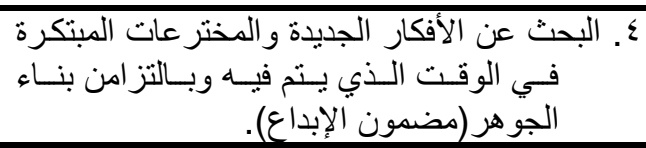 & ـ. التركيز على الإبداع ألتزايدي التدريجي. \\
\hline 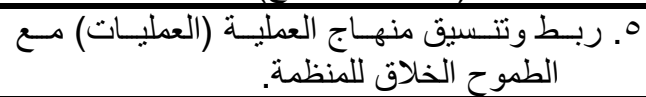 & إن \\
\hline 7. البحثت عن الاحتياجـات غير الظـاهرة(الضمنية) & 7. \\
\hline 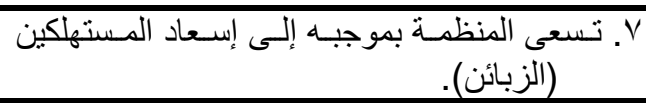 & $\mathrm{V}$ \\
\hline 1. قد يتم اختباره إز التيظيمية. المشاريع الجديدة والمغامرة & 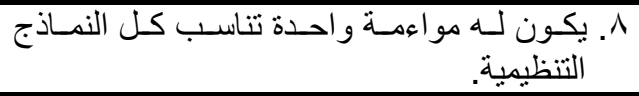 \\
\hline 9. الأفر اد الأذكيـاء داخل وخـارج المنظمـة مصدر & 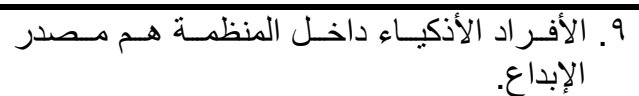 \\
\hline 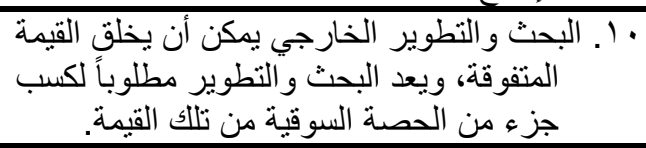 & 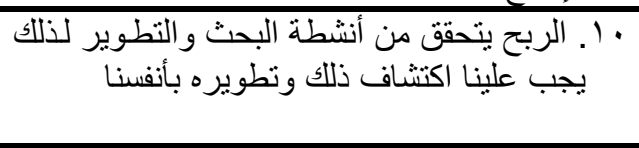 \\
\hline 11 ألجل علينا بالضرورة تشكيل البحث وتأهيله من & 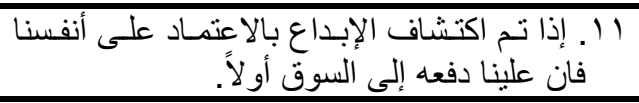 \\
\hline 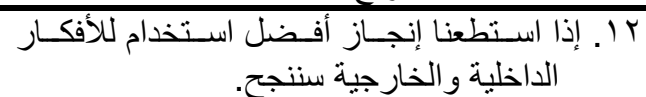 & 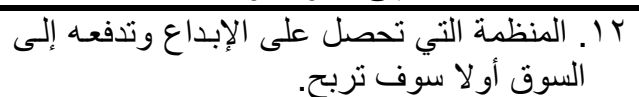 \\
\hline 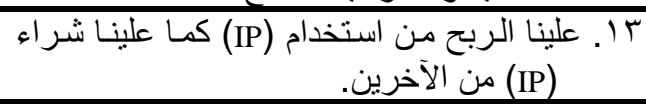 & 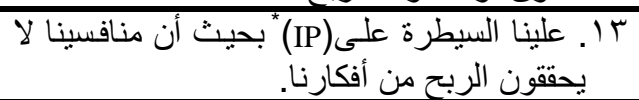 \\
\hline
\end{tabular}

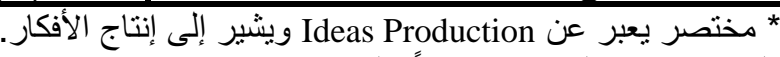
المصدر إعداد الباحثين إعتماداً على:

Source: Derrick Palmer and Soren Kaplan, (2007),Framework innovation, Blending strategy and Creativity to discover future business opportunities. (www. Innovation-point.com), p2.

- Soren Kaplan and Stu winby, (2007), Organizational Models for Innovation, Organizational Design that Support strategic Innovation and Growth .(www. Innovation-point.com), p9.

في إطار ما أفرزته العولمة من تغييرات منسار عة في الميادين الإدارية والاقتصادية

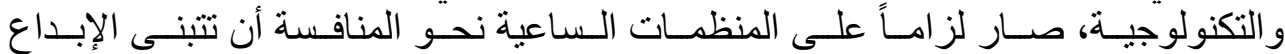

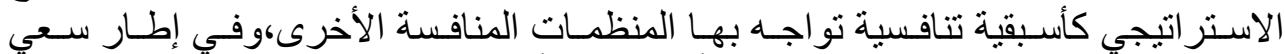

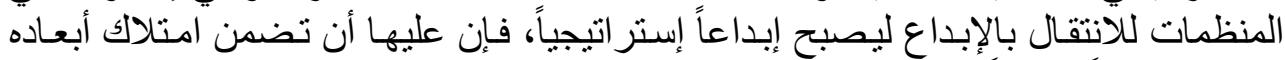
بوصفه شرطاً أساسياً لذلك ومن دون هذا الإمتلاك لاعت لا يمكن تحقيق الإبداع الاستر اتيجي.

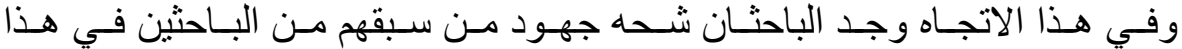

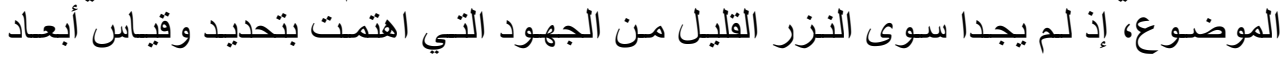

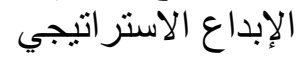

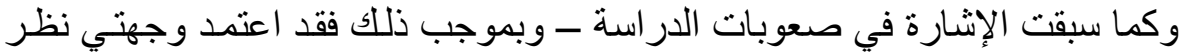

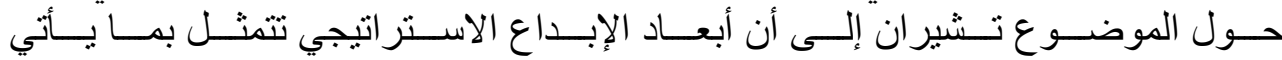

(Derrick and Soren, 2007, 5) و (www.innovation-point.com) 
أ. عملية الإبداع المدارة Managed Innovation Process

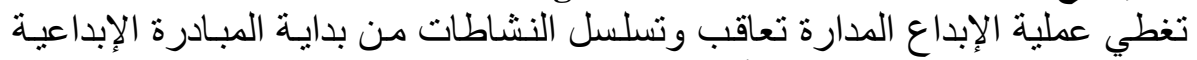

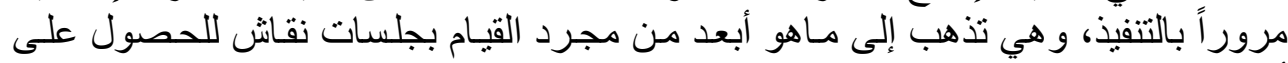

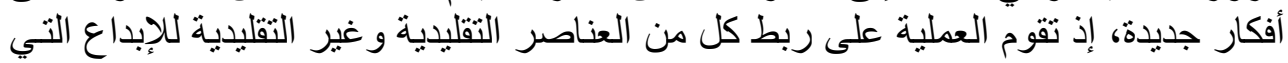

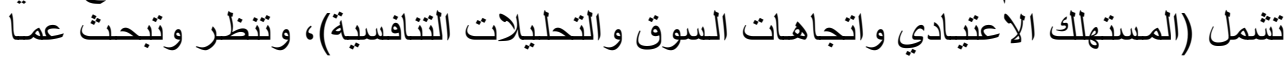

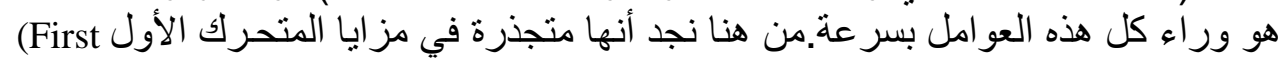
Mover Advantages)

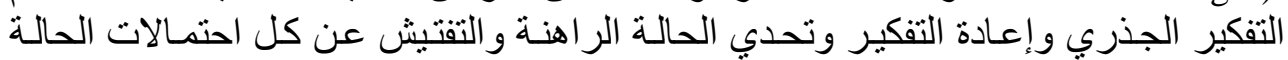

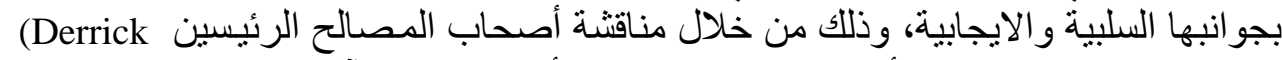

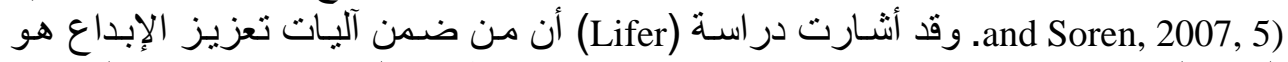

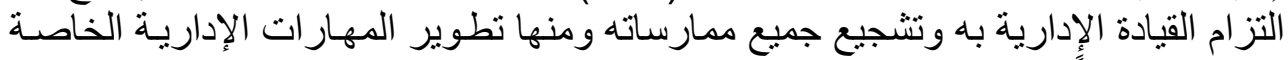

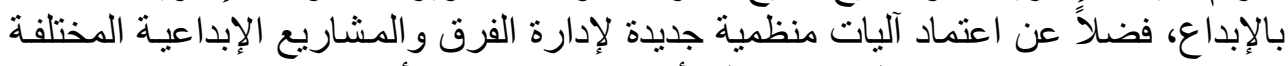

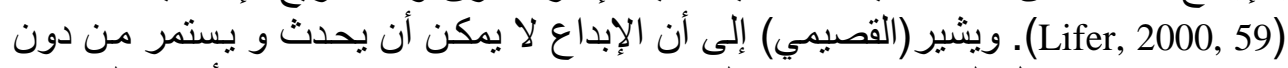

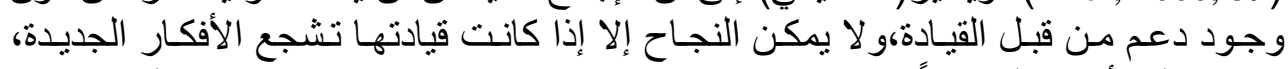

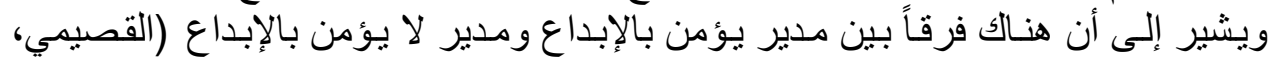

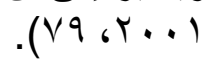

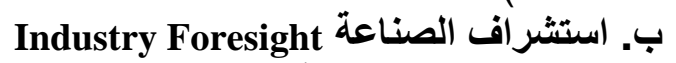

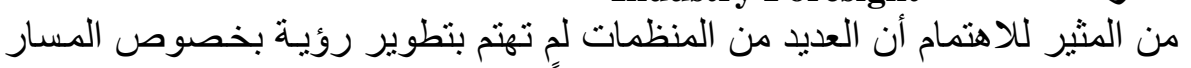

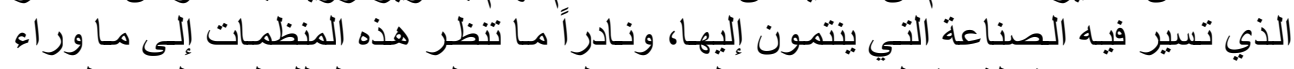

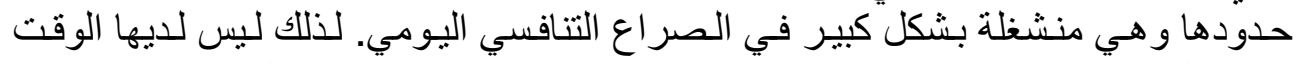

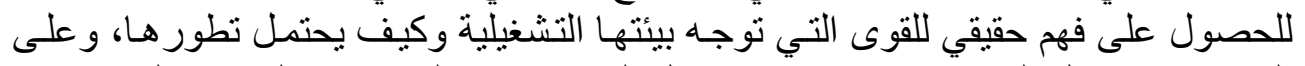

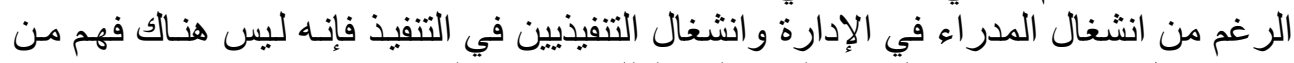

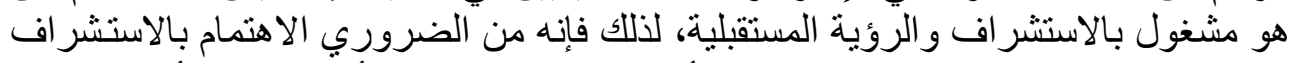

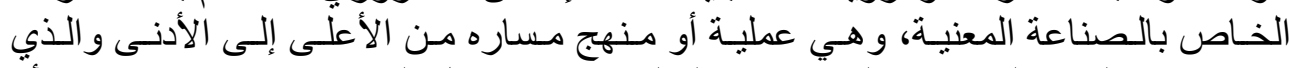

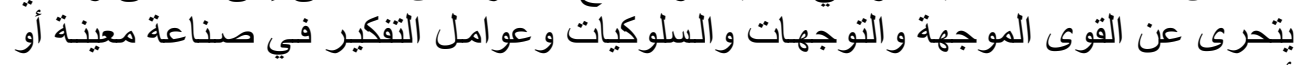

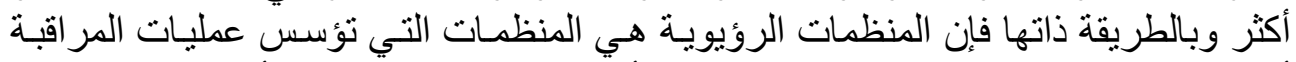

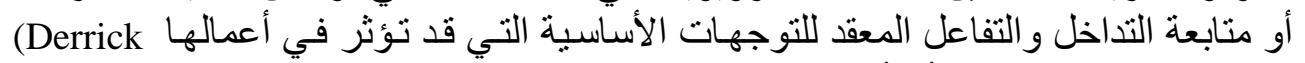
and Soren, 2007, 10)

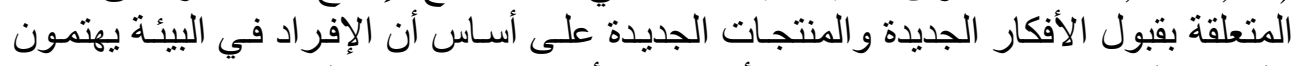

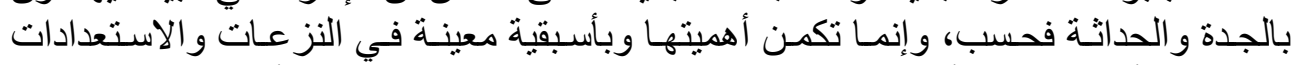

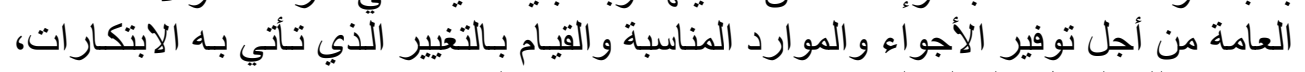

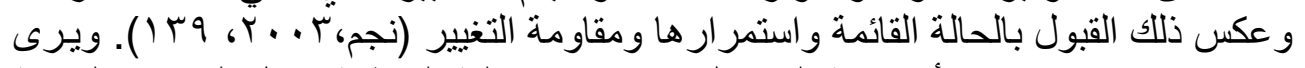

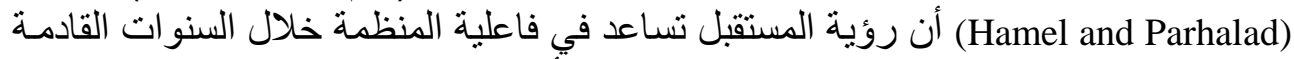

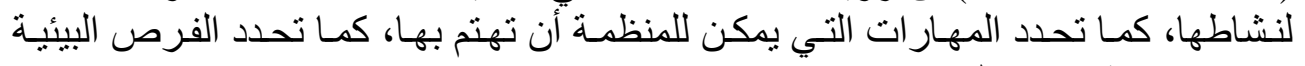
بوضوح ودقة لاستغلالها (Hamel and Parhalad,1999,127).

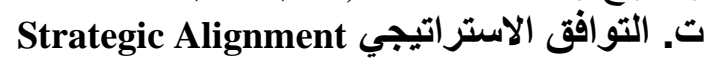

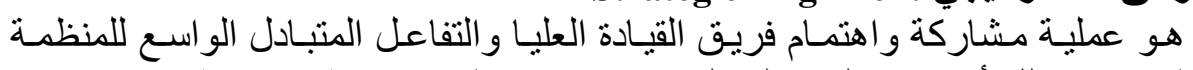

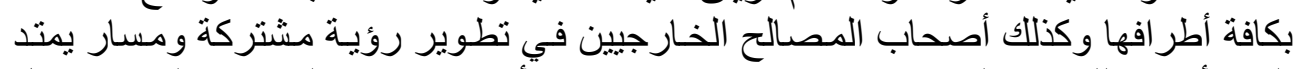

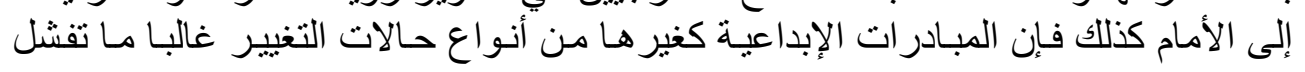




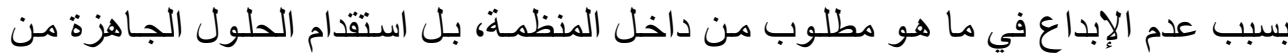

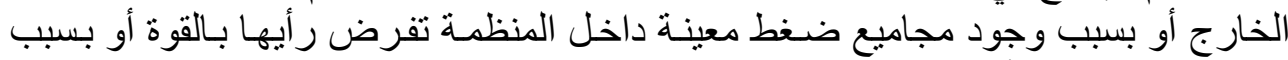

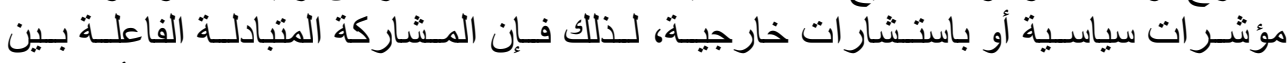

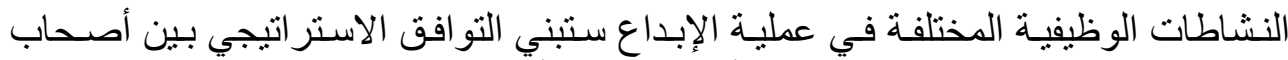

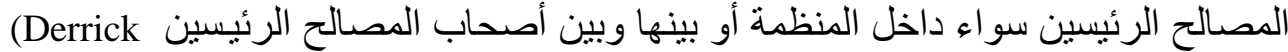
and Soren,2007,8)

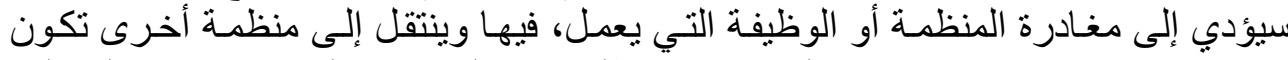

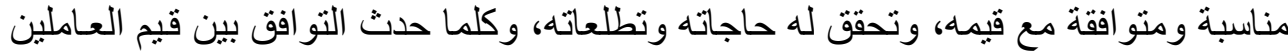

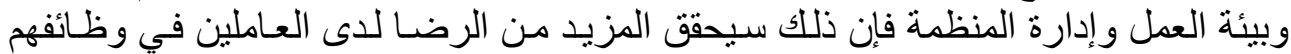

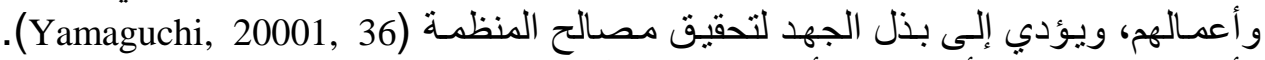

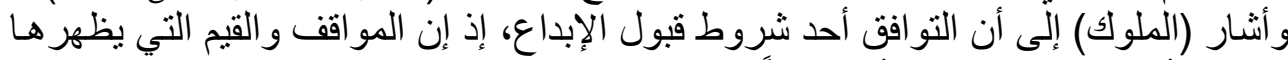

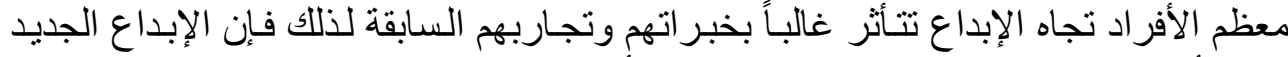

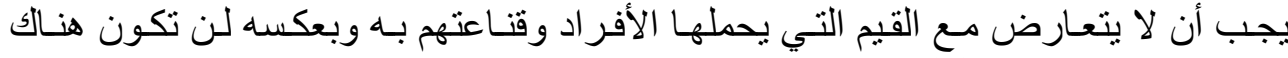

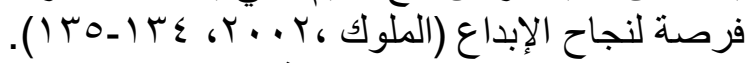

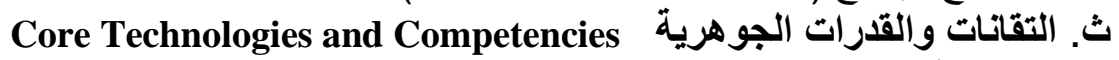

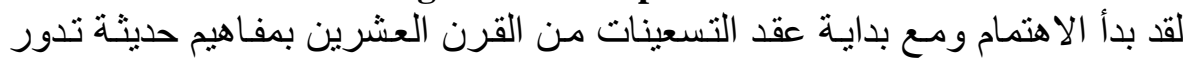

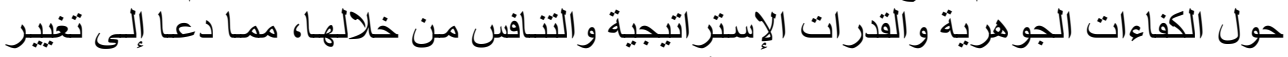

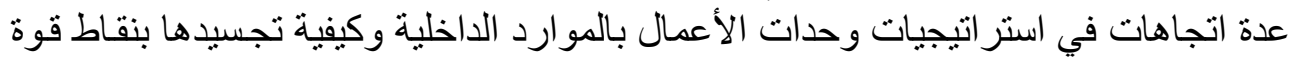

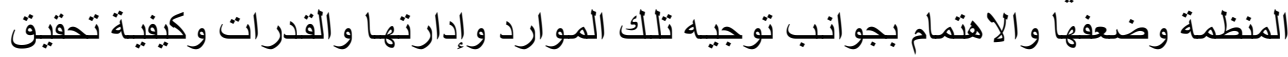

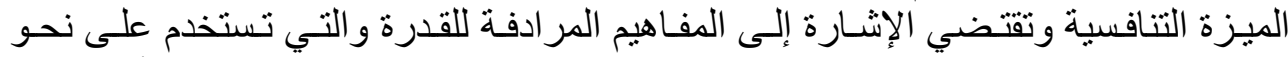

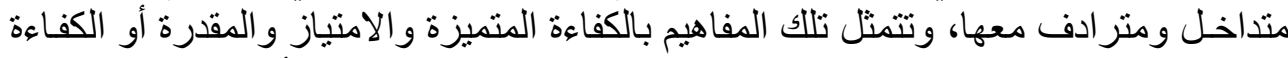

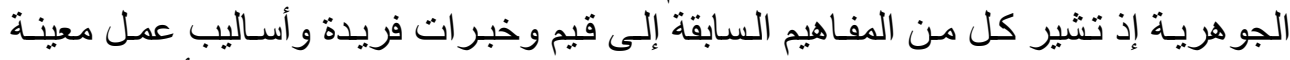

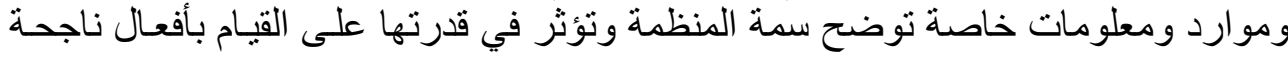

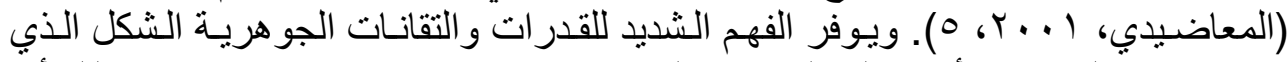

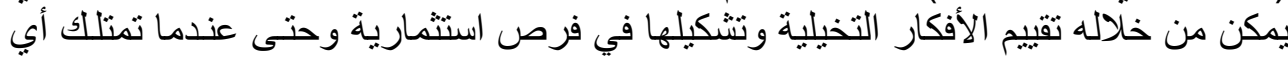

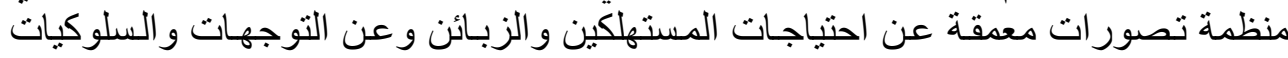

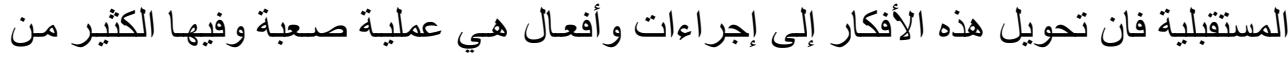

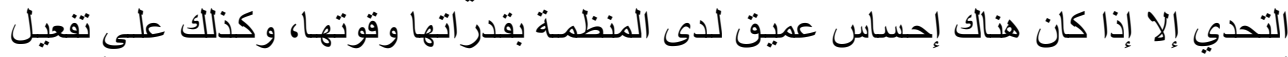

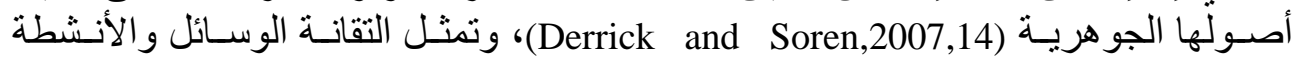

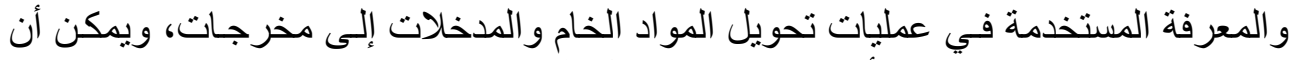

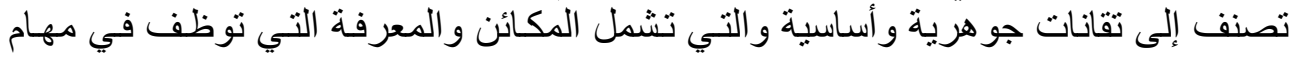

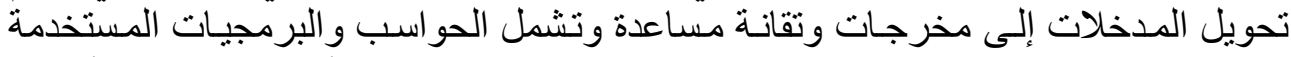

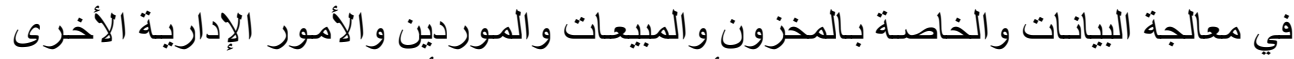

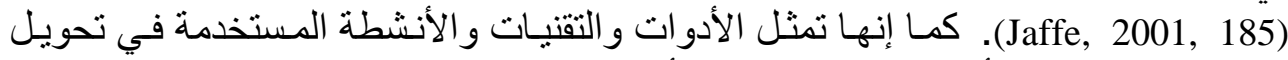

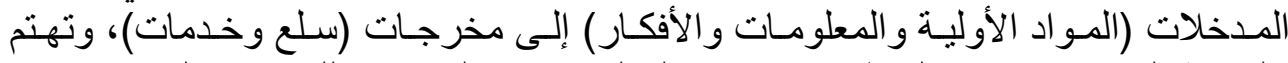

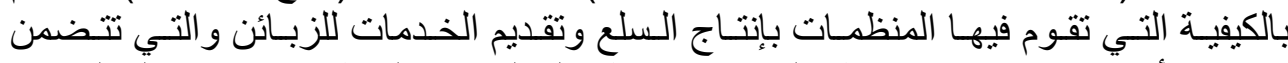

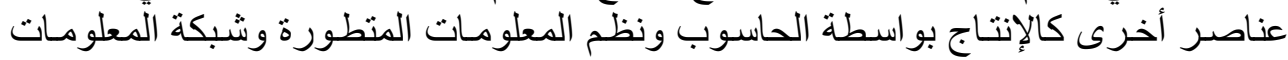

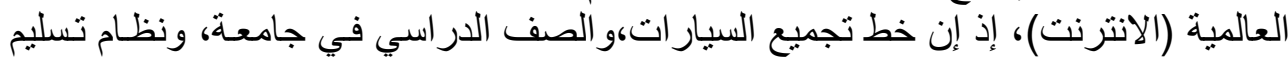


Daft, ) الطلبات للزبائن كلها تمثل تقانات، بـالر غم من اختلاف بعضها عن البعض الآخر (2001,199-200

\section{Customer Insight ث. تصورات الزيون}

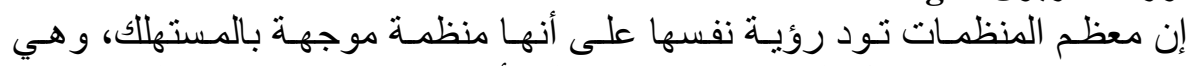

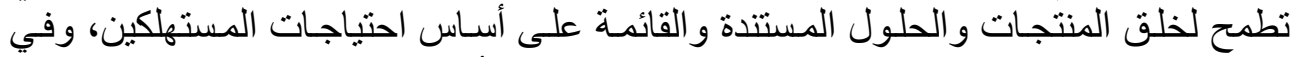

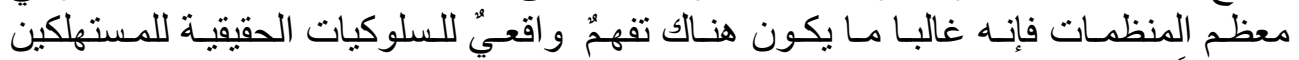

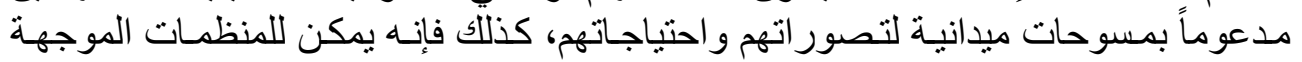

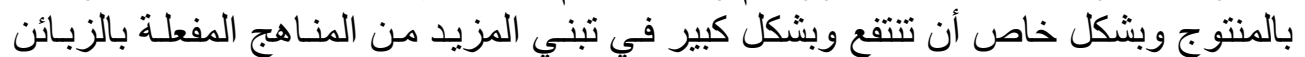

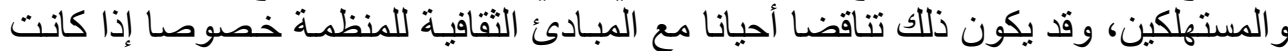

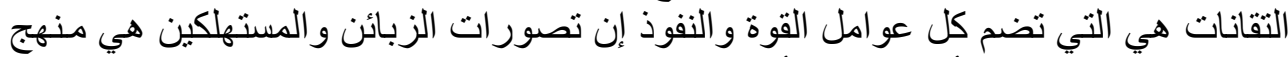

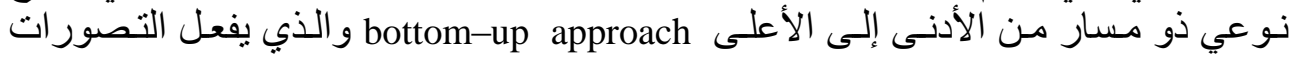

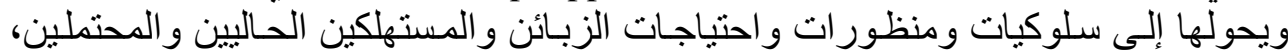

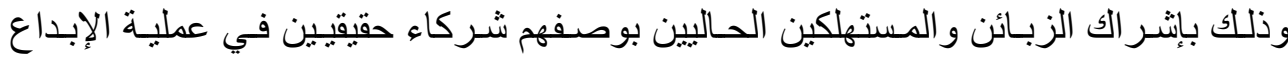

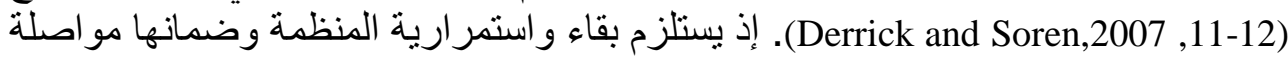

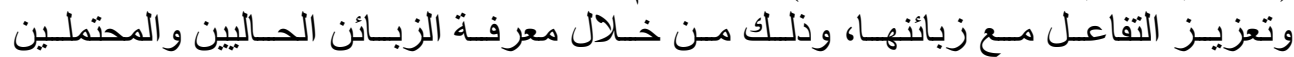

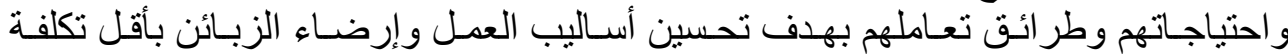

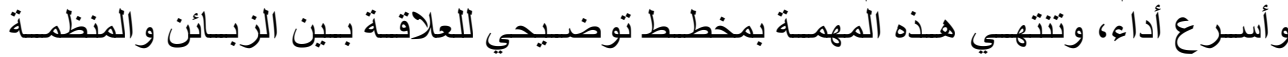

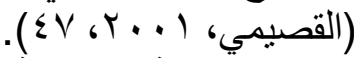

جrganizational Readiness الاستعدادية المنظمية

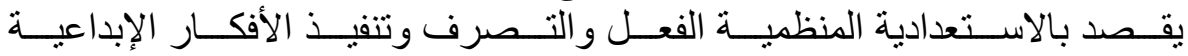

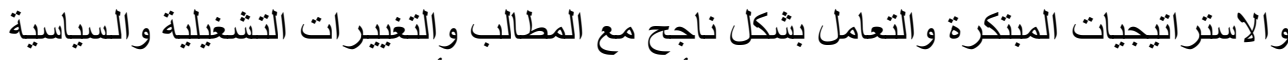

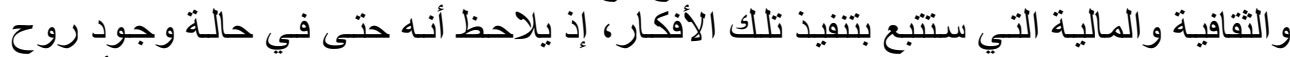

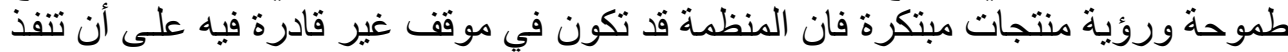

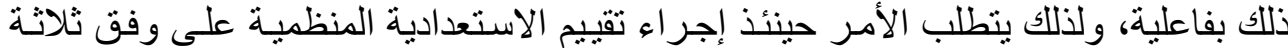

أبعاد (Derrick and Soren, 2007, 15). 
ـ الاستعدادية الثقافية. ـ الاستعدادية للعمليات. - الاستعدادية الهيكلية.

ا ـ الاستعدادية الثقافية

إن ثقافة المنظمـة (Corporate Culture) هي الاتجـاه الغالب أو السائد في المنظمـة المئة

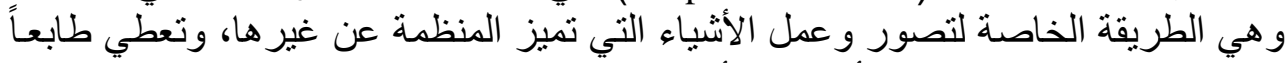

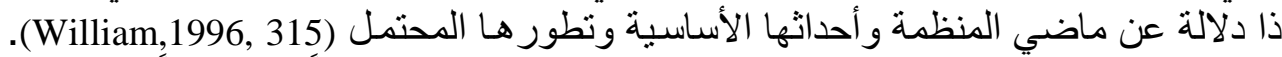

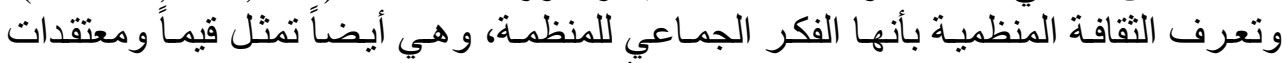

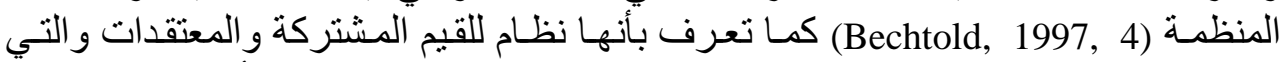

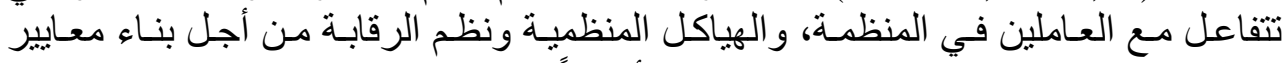

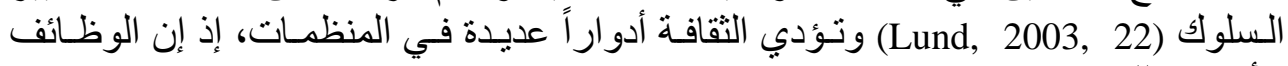

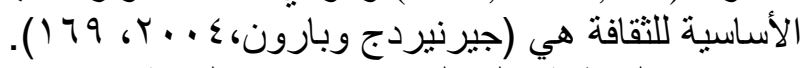

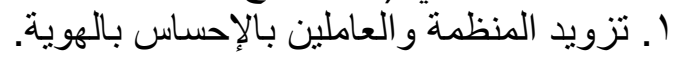

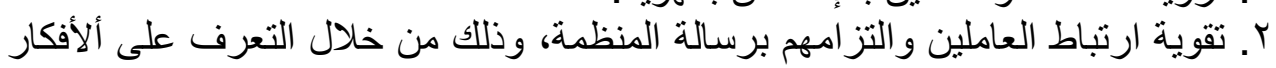
و القيم التي تسود في المنظمة التونة

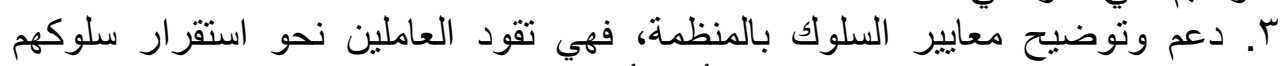

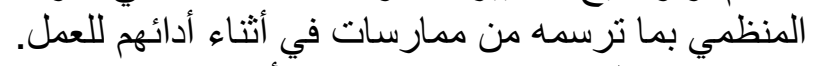

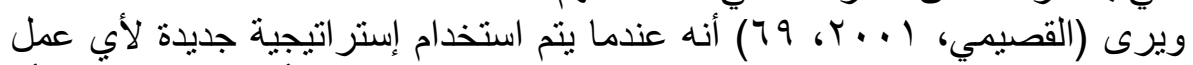

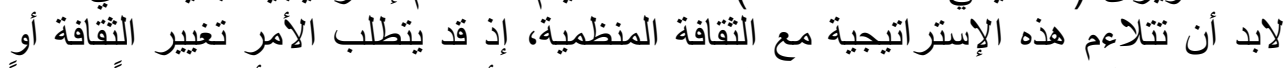

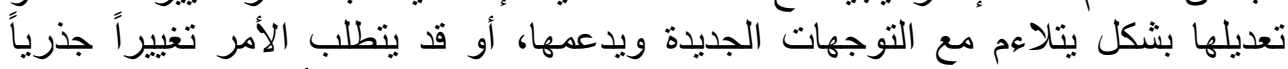

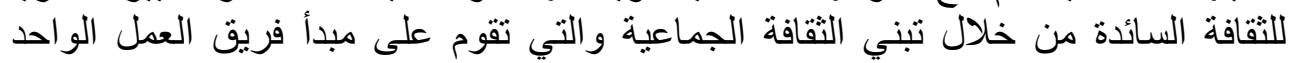

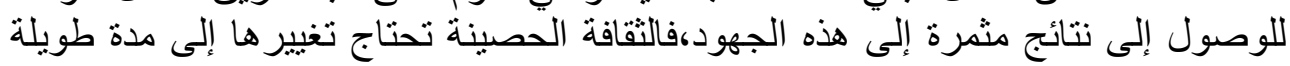

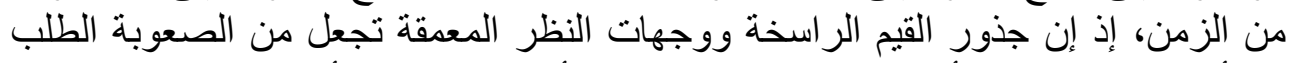

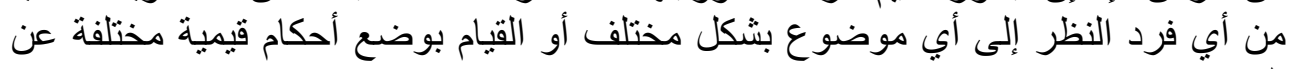

\section{r. الاستعدادية العملياتية}

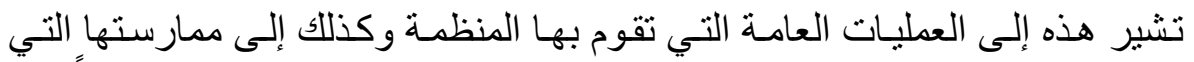

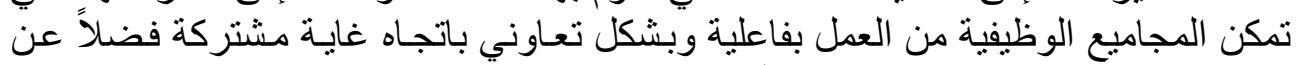

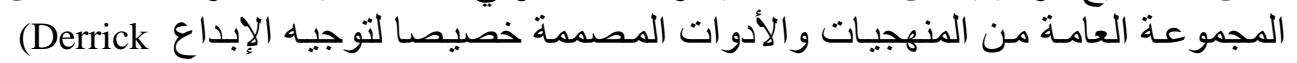
(and Soren, 2007, 15)

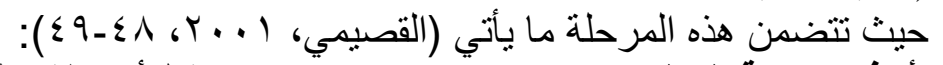

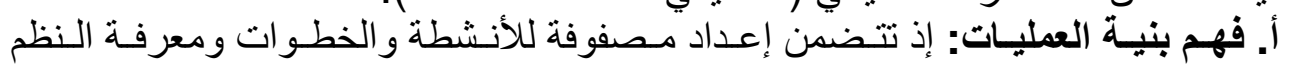

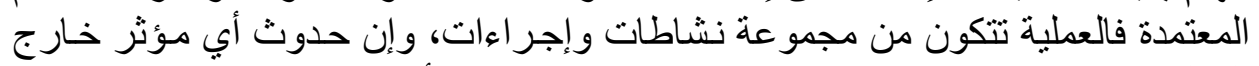

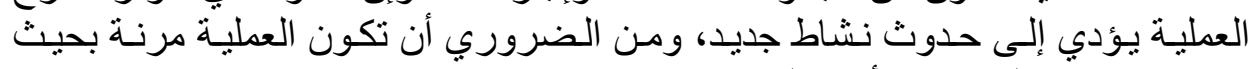
تستو عب جميع المتغير اتى حذوب أثناء التنفيذ. 


\section{المعاضيدي ومحمد] [111]}

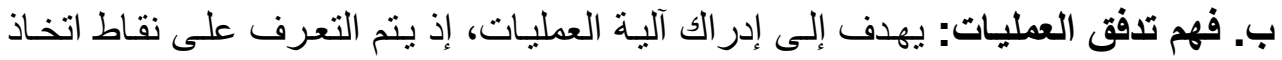

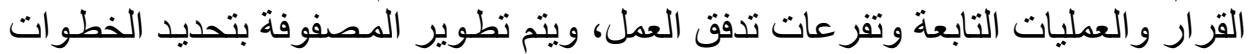

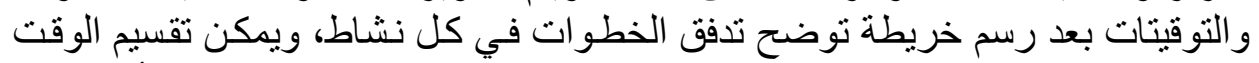

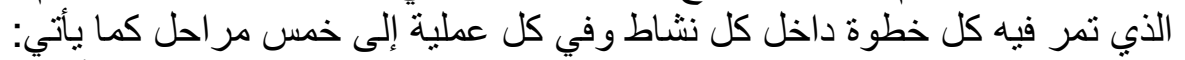
ا. وقت الانتقال: و هو الوقت الذي تستغرقه مدخلات النشاط حتى تصل إلى أول خطوة r. وقت انتظار المدخلات: ويفصل بين وصول المدخلات إلى نقطة العمل وبين بدء r. . وقت العمل الإجمالي: وهو الوقت الفاصل بين بدء العمل في المدخلات حتى بدء

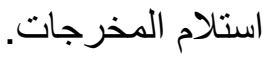
ع. ـ وقت العمل الصنافي: وهو الوقات الوقت الفعلي الذي أنجز فيه العمل ويكون دائماً مساوياً لوقت العمل الإجمالّي أو اقل منهـ. 0. وقت انتظار المخرجات: وهو الوقت الفاصل بين استكمال المخرجات وبدء انتقالها إلى نشاط جديد .

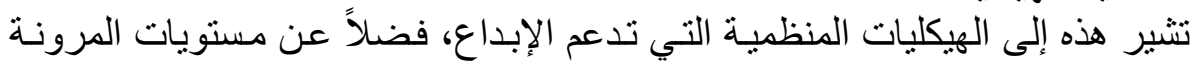

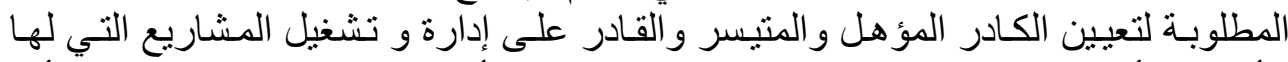

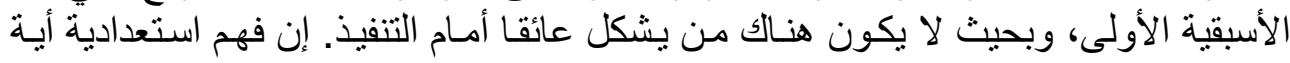

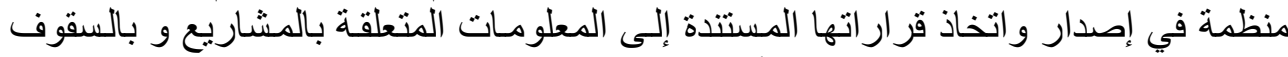

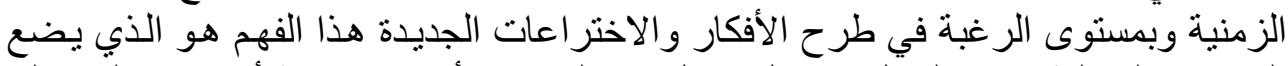

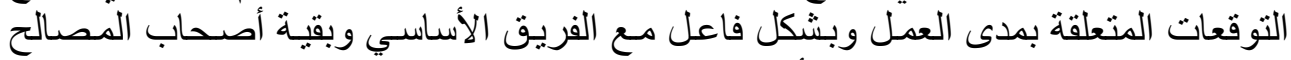
ويحقق إمكانية الوصول إلى منهج أو مدخل و اقعي للتنفيذ (Derrik and Soren, 2007, 15)

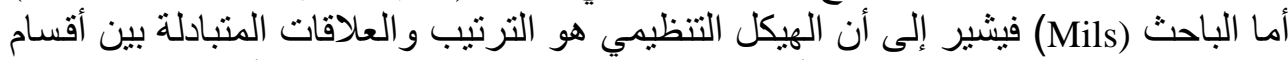

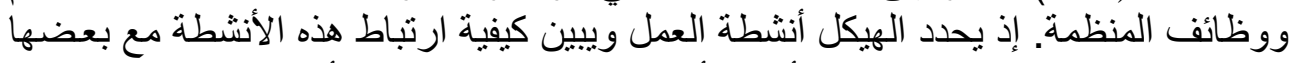

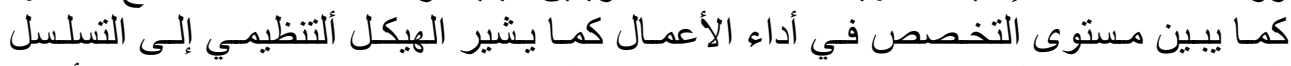

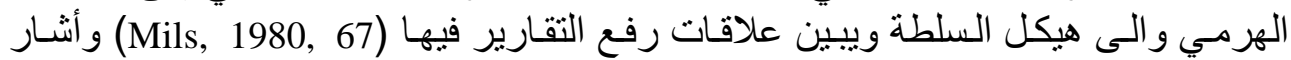

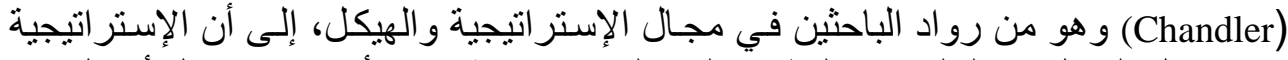

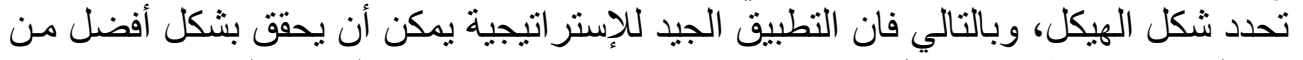

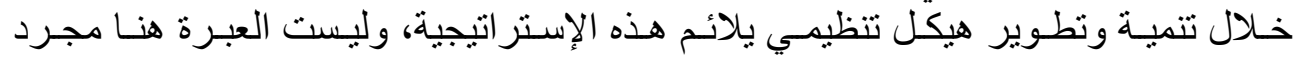

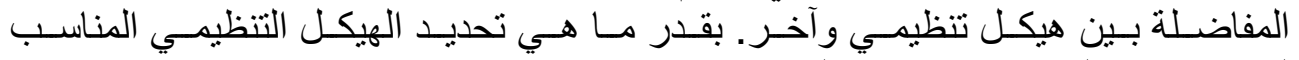

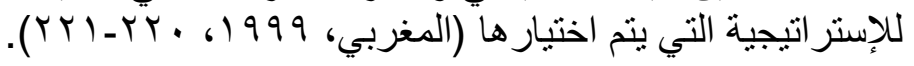

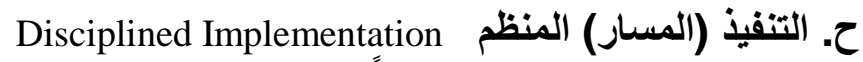

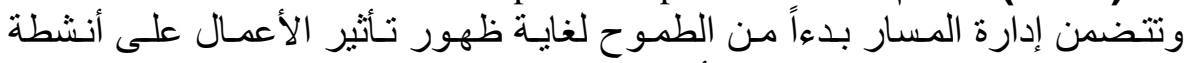

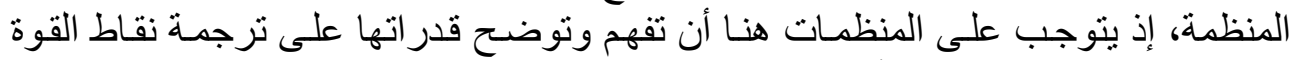

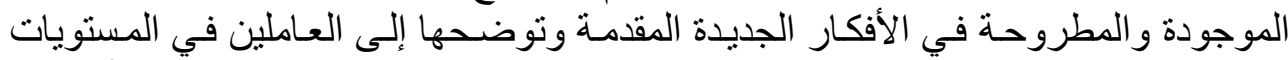

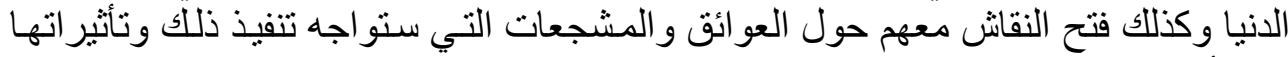

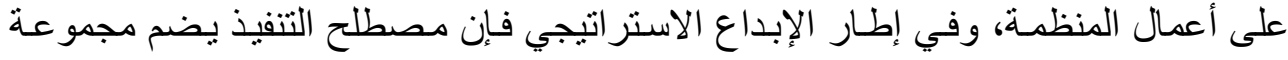




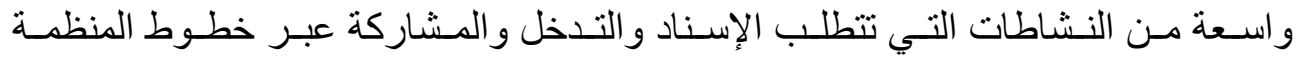

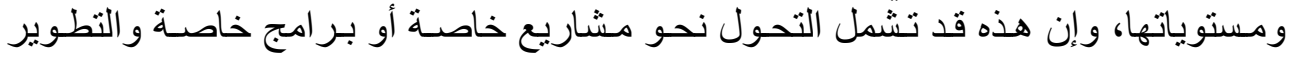

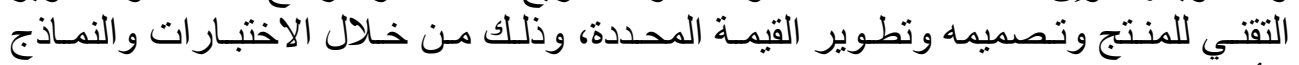

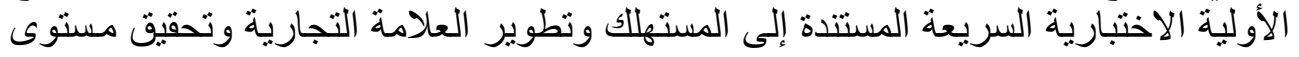

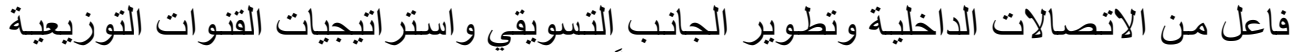

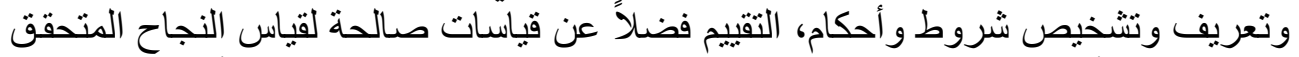

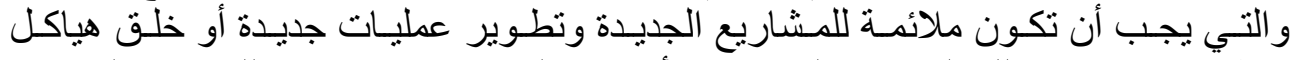

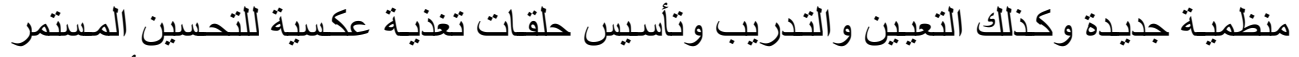
المتو اصل للعملية الإبداعية (Derrick and Soren, 2007,16) ويمكن توضيح هذه ونة الأبعاد من

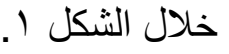

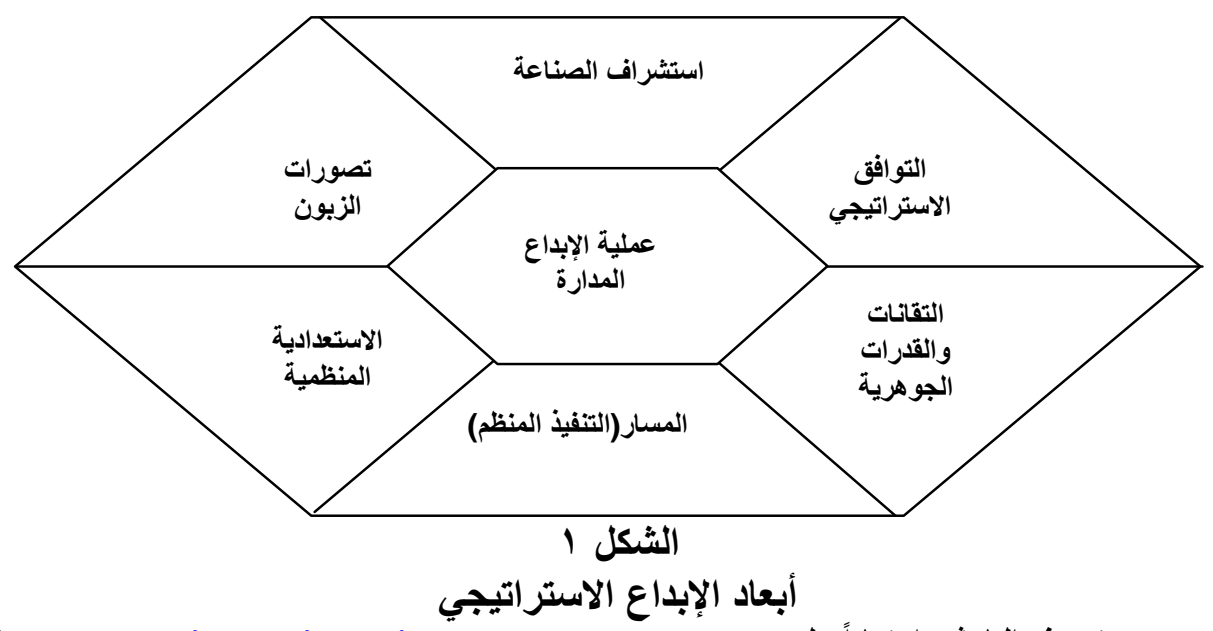

Source: www.innovation-point.com بتصرف الباحثين اعتماداً على :

الجاتب التطبيقي الأفراد المبحوثين

اتسمت عينة البحث وفقاً للبيانات التي قدمها أفرادها من خلال إجاباتهم عن الجزء الجئ

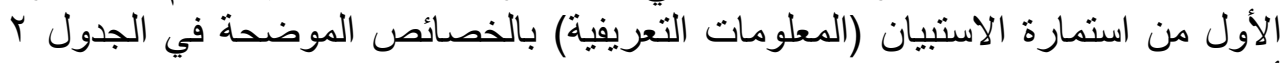

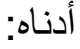




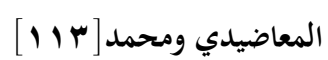

توزيع أفراد عينة البحث بحسب بعض الخصائص

\begin{tabular}{|c|c|c|c|c|c|c|c|c|c|c|c|c|c|}
\hline \multicolumn{14}{|c|}{ المركز الوظيفي } \\
\hline \multicolumn{4}{|c|}{ مسؤول وحدة } & & \multicolumn{4}{|c|}{ مدير شعبة } & & \multicolumn{4}{|c|}{ رئيس قسم } \\
\hline \multicolumn{2}{|c|}{$\%$} & \multicolumn{2}{|c|}{ العدد - العد } & & \multicolumn{2}{|c|}{$\%$} & \multicolumn{2}{|c|}{ 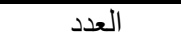 } & & \multicolumn{2}{|r|}{$\%$} & \multirow{2}{*}{\multicolumn{2}{|c|}{ العدد }} \\
\hline & & & $r$ & & & & $\bar{v}$ & & & & 0.0 & & \\
\hline \multicolumn{14}{|c|}{ الجنس } \\
\hline \multicolumn{7}{|c|}{ إناث } & \multicolumn{7}{|c|}{ ذكور } \\
\hline & & \multicolumn{2}{|c|}{$\%$} & \multirow{2}{*}{\multicolumn{3}{|c|}{ 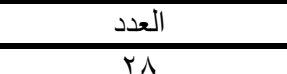 }} & \multirow{2}{*}{\multicolumn{3}{|c|}{$\%$}} & \multicolumn{2}{|r|}{ العدد } & & \\
\hline & & \multicolumn{2}{|c|}{ ro.v } & & & & & & & & 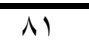 & & \\
\hline \multicolumn{14}{|c|}{ التحصيل العلمي } \\
\hline \multicolumn{3}{|c|}{ إعدادية } & \multicolumn{2}{|c|}{ دبلوم د } & \multicolumn{2}{|c|}{ بكالوريوس } & \multicolumn{2}{|c|}{ دبلوم عالِ } & \multicolumn{2}{|c|}{ ماجستير } & \multicolumn{3}{|c|}{ دكتوراه } \\
\hline & & العدد العد & $\%$ & العدد & $\%$ & العدد & $\%$ & لعدد ل لعد ال & $\%$ & عدد & $\%$ & & العدد \\
\hline & & 1. & $1 T . V$ & 10 & .79 & $V_{7}$ & $\ldots$ & $\ldots$ & V.r & $\Lambda$ & ... & & $\ldots$ \\
\hline \multicolumn{14}{|c|}{ الفئات العمرية } \\
\hline \multicolumn{3}{|c|}{. م فأكثر } & \multicolumn{2}{|c|}{$\leqslant 9-\leqslant 0$} & \multicolumn{2}{|c|}{$\varepsilon \varepsilon-\varepsilon}$. & \multicolumn{2}{|c|}{ rq-ro } & \multicolumn{2}{|c|}{$r \varepsilon_{-} r}$. & \multicolumn{3}{|c|}{ rq- ro } \\
\hline & & العدد & $\%$ & العدد & $\%$ & العدد & $\%$ & لعدد ل ل ل & $\%$ & عدد & $\%$ & & العدد ال العد \\
\hline & & $\Lambda$ & R.r & 9 & 11 & $\overline{T r}$ & $r \mu . \Lambda$ & rT & $\overline{r . . V}$ & $\overline{r^{9}}$ & $\overline{Y Y}$ & & ro \\
\hline & & & & & & اخذمة & & & & & & & \\
\hline & & & $\frac{14}{T 4}$ & & & & 17 & & & T. & & ل فأقّل & o \\
\hline$\%$ & 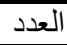 & $\%$ & 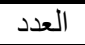 & $\%$ & العدد & $\%$ & العدد & $\%$ & العدد & $\%$ & العدد & $\%$ & العدد العد الع \\
\hline$\overline{r . v V}$ & $r$ & $V . \varepsilon$ & $\Lambda$ & $\ldots$ & $\ldots$ & $\pi$ & $\overline{T r}$ & $\pi$ & $T r$ & $r r .9$ & $r v$ & $r r .9$ & $r v$ \\
\hline
\end{tabular}

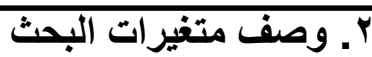

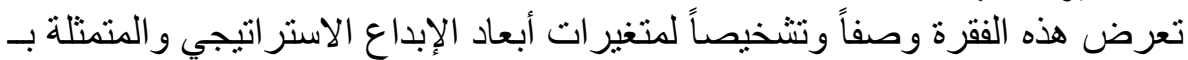

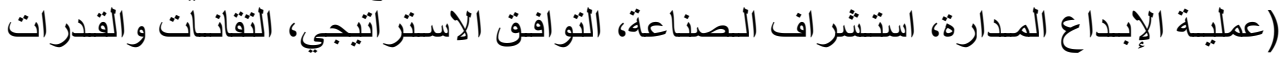

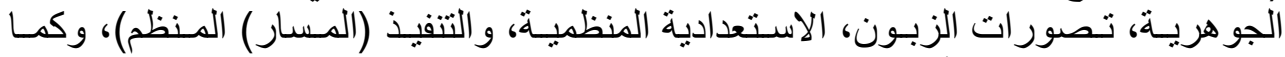

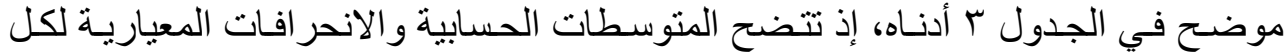

متغير رئيس وفر في للمتغير ات التي شملها البحث. 
التكرارات والنسب المئوية والتوزيعات التكرارية والاتحر افات المعيارية لمتغيرات البحث

\begin{tabular}{|c|c|c|c|c|c|c|c|c|c|c|c|c|c|}
\hline \multirow{2}{*}{ معرلف } & \multirow{2}{*}{ سمائ } & \multicolumn{2}{|c|}{ لا اتثلى بُشدة } & \multicolumn{2}{|c|}{ لا اتفق } & \multicolumn{2}{|c|}{ بديا } & \multicolumn{2}{|c|}{ النقى } & \multicolumn{2}{|c|}{ تفئ بيدة: } & \multicolumn{2}{|c|}{ المثظبرات } \\
\hline & & $\%$ & 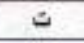 & $\%$ & $\Xi$ & $\%$ & 0 & $\%$ & $\Delta$ & $\%$ & 0 & فز & رلمبسي \\
\hline .,krqQi & $r, 2 r+4$ & $1, \mathrm{~A}$ & $r$ & $r, \mathrm{Y}$ & $i$ & $r A, 0$ & it & $i r, Y$ & in & $1 \pi, \lambda$ & 10 & $\mathrm{X}_{1}$ & \multirow{8}{*}{$\begin{array}{l}\frac{3}{3} \\
\frac{7}{4} \\
\frac{7}{3} \\
\frac{7}{6}\end{array}$} \\
\hline - ArTIA & $r, 00.0$ & $1, A$ & $r$ & $\gamma, T$ & $\mathrm{~A}$ & $r T, 9$ & rv & $\{\mathrm{v}, \mathrm{V}$ & or & $9, \uparrow$ & 1. & $\mathrm{X}_{2}$ & \\
\hline, 99077 & $r, t 0 \mathrm{NV}$ & $1, \mathrm{~A}$ & 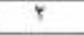 & $1 \mathrm{t}, \mathrm{V}$ & 18 & $P 0, A$ & $r 4$ & $r 1, r$ & $T f$ & 19,0 & in & $\mathrm{X}_{3}$ & \\
\hline$\because 9 \wedge q+\vee$ & r,AOTY & $1, \mathrm{~A}$ & $r$ & 7,8 & $\mathrm{v}$ & 79,9 & $r^{2}$ & $r \varepsilon, 9$ & rA & $r \cdot, r$ & rr & $X_{4}$ & \\
\hline - Y,YA.Y1 & $r, Y Y \leqslant A$ & $\bar{E}$ & $=$ & $Y_{,}, \mathrm{r}$ & 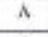 & $40, Y$ & $r \mathrm{~A}$ & $0 \leqslant, 1$ & 09 & $19, \hat{A}$ & If & $X_{s}$ & \\
\hline $0.940 . \mathrm{V}$ & r.,OAYY & $r, A$ & + & 3,2 & $\checkmark$ & $r 0,1$ & $r 4$ & $r 4,2$ & is & 10,3 & iv & $X_{6}$ & \\
\hline $1, \cdot 1 . v r$ & rilaro & $r, Y$ & f & re & Yi & rq,y & 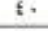 & $r v, 0$ & $r$. & $1 . .1$ & 11 & $\times 7$ & \\
\hline \multirow[t]{2}{*}{$1, .2737$} & r,orra & $r, V$ & f & $m$ & 14 & $r v, 7$ & 5 & $x \leqslant, \wedge$ & zi & $r Y, q$ & Yo & X8 & \\
\hline & $r, 0 \pi r$. & $Y, 1$ & & 9,10 & & & & ry, it & & 17.8 & & \multicolumn{2}{|c|}{ الall } \\
\hline Y9Y9/A & $r, 9051$ & .9 & 1 & $r, \AA$ & $r$ & 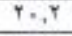 & re & Or,, $\mathrm{r}$ & ov & $r+q$ & Y9 & $X_{8}$ & \\
\hline A0VY4 & $r, 00.0$ & $1, \AA$ & ${ }^{\top}$ & $14, \hat{A}$ & 12 & $\mathrm{YA}, \mathrm{i}$ & ri & $E Y, Y$ & 29 & $1 \mathrm{f}, \mathrm{Y}$ & 17 & $\mathrm{X}_{10}$ & it \\
\hline 1.0 .0$. & r,orr) & $r, \mathrm{~V}$ & \& & $1 \%, A$ & 15 & $\mathrm{TA}, \mathrm{s}$ & ri & $r+, v$ & 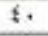 & $1, k, r$ & $r$. & $\mathrm{X}_{11}$ & g \\
\hline $1,+\varepsilon<5$. & T.rरרI & 6.7 & $\circ$ & $1 \pm, Y$ & 17 & $T T$ & Ti & $r+.4$ & rv & $1 \Gamma, A$ & 10 & $X_{12}$ & 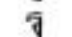 \\
\hline $.901+7$ & $r, 1 Y \leq r$ & $\gamma, \hat{A}$ & $r$ & $\pi, 9$ & To & $r t .9$ & TA & $\pi$ & $r 1$ & 7,8 & Y & $\mathrm{Xl}_{3}$ & \\
\hline \multirow[t]{2}{*}{.80711} & rivrea & $=$ & - & 7,1 & $\mathrm{v}$ & 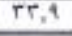 & rv & $r 4,2$ & iT & $r \cdot, r^{r}$ & rT & $\mathrm{XI}_{4}$ & \\
\hline & r,00ro & $T, T$ & & $19, \cdot 7$ & & & & $r 9,0 \mathrm{~A}$ & & $17, \pi 1$ & & \multicolumn{2}{|c|}{ القعتل } \\
\hline 92191 & r, riar & 1,1 & r & 18,7 & 19 & $r A, 0$ & $\xi \gamma$ & $\pi$ & ri & 11,9 & iT & $\mathrm{X}_{15}$ & \\
\hline 1,19471 & $r, \pi+11$ & $Y, T$ & $\lambda$ & $M, r$ & Y. & YO,Y & YA & $r Y, 1$ & ro & 17,0 & in & $X_{16}$ & 7 \\
\hline \multirow[t]{2}{*}{$.94 \times 4}$. & $r, T+i, 1$ & $r, v$ & $i$ & $1 \mathrm{~V}, \mathrm{z}$ & 19 & TY,1 & ro & $r q, y$ & ક. & $\cdots$, & 11 & $\mathrm{X}_{17}$ & \\
\hline & $r, r \in \times 0$ & $f, Y$ & & 17,1 & & & & $r r, \cdot r$ & & $1 T, A r$ & & \multicolumn{2}{|c|}{ المعلd } \\
\hline $9 \pi \times 11$ & $r, 3: \mathrm{tr}$ & $r, \Lambda$ & $r$ & $r, \mathrm{~V}$ & $\varepsilon$ & $\{\cdot, \hat{\imath}$ & $\varepsilon \mathrm{i}$ & $\pi$ & rq & $\varphi^{\mu,,^{4}}$ & YY & $\mathrm{X}_{18}$ & \multirow{5}{*}{ 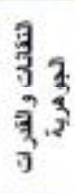 } \\
\hline .9Y TVर & T, TiA1 & $r, \AA$ & $r$ & 11,9 & $i r$ & it & iA & $r \cdot, r$ & rt & 11 & 17 & $\mathrm{X}_{19}$ & \\
\hline $1, .57=1$ & r.६90\} & $\{, 7$ & 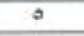 & 9,7 & 1. & $r_{0, A}$ & T4 & $T r$ & ry & $Y, i$ & 14 & $\mathrm{X}_{20}$ & \\
\hline $1,+Y \leqslant Y \leqslant$ & r.trr. & $r, 1$ & $T$ & 11,9 & $i 5$ & $r i, A$ & TV & $t, t$ & ti & $r \cdot,^{\top}$ & TT & $X_{21}$ & \\
\hline \multirow[t]{2}{*}{$1,+7 v \cdot v$} & $r, \$ \$ 40$ & $4, \lambda$ & $r$ & 13,0 & IA & $r+4$ & $T$ & $\gamma \Lambda, f$ & TI & 19.7 & 71 & $\mathrm{X}_{22}$ & \\
\hline & $r, 01, r y$ & $r, 1$ & & $1,7 \mathrm{i}$ & & & & $T r, \cdot T$ & & IV,TY & & & \\
\hline - Arqo & $5,74 \times 9$ & $1, A$ & $T$ & $T, Y$ & $!$ & $\Gamma \mu_{1}, 0$ & iT & $i T, Y$ & 27 & 15,1 & 10 & $X_{23}$ & \\
\hline - ATTIA & $5,00.0$ & $1, \AA$ & $\gamma$ & $V, r$ & $A$ & $r+1$ & TV & $f \mathrm{f}, \mathrm{V}$ & or & $4, T$ & 1. & $X_{24}$ & 2 \\
\hline 0,99017 & $r, t e \times v$ & $1, \AA$ & $x$ & $1 \mathrm{f}, \mathrm{V}$ & 17 & $r_{0, \lambda}$ & $r 4$ & $r, r^{r}$ & ri & 17,0 & $i x$ & $X_{25}$ & ง \\
\hline$\cdot, 4 \lambda 1, \gamma$ & r.Aotr & $1, A$ & $r$ & 7,8 & $\mathrm{v}$ & 77,7 & $\pi$ & $r i, 4$ & TA & $T \cdot, T$ & $\pi$ & $X_{26}$ & \\
\hline$=, \times A,+1$ & $r, V Y \in A$ & $\bar{E}$ & & $\gamma, r$ & $\mathrm{~A}$ & $r o, v$ & TA & $\Delta i, 1$ & 09 & $19, A$ & if & $\mathrm{x}_{27}$ & ja \\
\hline $0,940,4$ & $r, 0 \wedge V Y$ & $Y, A$ & $r$ & 7,2 & $\mathrm{v}$ & $r 0, A$ & rq & $r 4,8$ & sr & 10,1 & iv & $x_{38}$ & \\
\hline & $r, \varepsilon 71 \mathrm{~V}$ & 7,7 & & $11,4 \pi$ & & & & $r 1,9$. & & $10, \sqrt{ }$ & & & \\
\hline 96174 & $r, r+1$, & $T, Y$ & if & $18, \AA$ & 18 & $4 \cdot, 4$ & it & $r T, 4$ & TV & 9,4 & 1. & $\mathrm{X}_{29}$ & \\
\hline ATeOr & $r, 5119$ & 9 & 1 & 17,0 & 11 & $r v, 7$ & f) & $\varepsilon \cdot, \varepsilon$ & if & 6,7 & - & $X_{30}$ & \\
\hline 1.9901 & r.riाa & $1, \mathrm{~A}$ & $T$ & 10,7 & iv & $f \cdot, f$ & it & $r \pi, 1$ & Tr & $A, T$ & 9 & $X_{31}$ & 3 \\
\hline A. $00: T$ & 5,1901 & $1, \AA$ & $r$ & $r \cdot, r$ & ri & $11, \pi$ & to & rT & ri & $r, v$ & ؛ & $x_{32}$ & \\
\hline $1,1+4 .$. & $r, 17 \mathrm{Af}$ & 0,0 & 7 & $T \mathrm{Y}, 0$ & $r$. & $Y A, i$ & 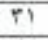 & Yo,v & TA & $17, \hat{A}$ & $1 \mathrm{t}$ & $X_{43}$ & 3 \\
\hline 1,1.Ar. & $5,11,1$ & $9, \%$ & 1. & $1, A$ & r. & $r \varepsilon, 9$ & Th & $\mathrm{YV}, \mathrm{O}$ & $r$. & 1,1 & 11 & $X_{44}$ & 3 \\
\hline 1, . Ter & $r_{1} \cdot \mathrm{vrf}$ & 9,6 & $Y$ & $r, r$ & rT & $f=, \hat{f}$ & $\{!$ & $r 0, \mathrm{~V}$ & rA & v,r & A & $X_{15}$ & 3 \\
\hline $1,191 \times 5$ & Y, vrra & 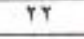 & $Y i$ & $1 \mathrm{f}, \mathrm{Y}$ & 17 & $r \times, 7$ & 4 & $19, \pi$ & ri & $7, \varepsilon$ & $\mathrm{Y}$ & $X_{36}$ & \\
\hline $.94 \wedge \leqslant \mathrm{V}$ & $r, .00$. & $y, r$ & $\mathrm{~A}$ & 17,0 & 11 & $f Y, Y$ & or & $r \cdot, T$ & YY & $A, r$ & 9 & $\mathrm{X}_{12}$ & \\
\hline & r, 1 rio & 7,0 & & $1,1, r$ & & & & $T \wedge, \wedge \varepsilon$ & & $8, \wedge 0$ & & & \\
\hline .98 .90 & r, rnor & $r, A$ & $r$ & 18,8 & 11 & TY,O & r. & $\varepsilon \uparrow .1$ & \&V & 9,7 & 1. & $\mathrm{X}_{18}$ & \\
\hline $1, .7979$ & r.rvרl & 0,0 & 7 & 10,7 & iv & $Y Y, 0$ & $r$. & $\mathrm{TA}_{\mathrm{N}} \mathrm{O}$ & it & $17, A$ & $1 E$ & $\mathrm{X}_{39}$ & 電： \\
\hline ATTYA & $r, r \cdot r \Lambda$ & 9 & 1 & $15, \wedge$ & 10 & 19,1 & 01 & $r 1, T$ & ri & $\gamma, T$ & $\mathrm{~A}$ & $X_{40}$ & \\
\hline 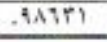 & $r, 101 T$ & $T, V$ & $\mathrm{f}$ & 1,1 & 11 & $T 9,6$ & rx & $E Y, Y$ & $\$ 1$ & $14, Y$ & 17 & $X_{41}$ & \\
\hline & $r, t, 1 T$ & $T, T$ & & $16, X Y$ & & & & $\left.r \lambda_{1}\right\rangle_{0}$ & & 11 & & & \\
\hline
\end{tabular}




\section{[110] المعاضيدي ومحمد}

بهدف التعرف على طبيعة العلاقة بين أبعاد الإبداع الاستراتيجي، ومدى تو افر ها في

المنظمة المبحوثة فقد اعتمد على مجمو عة من الأدوات التحليلية الإحصائية التي تم ذكر ها.

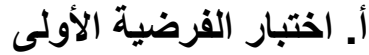

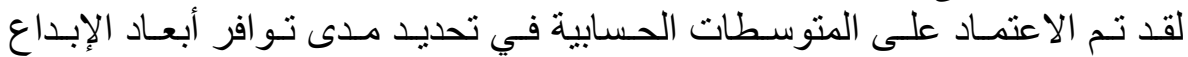

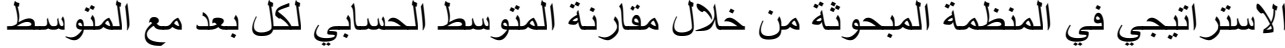

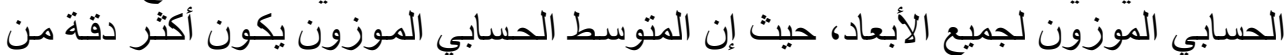

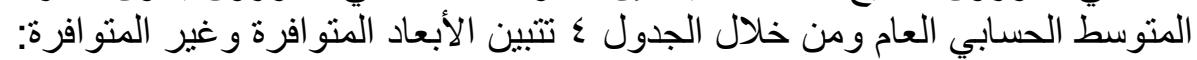

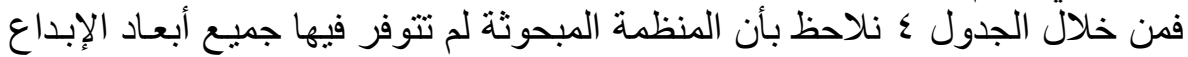

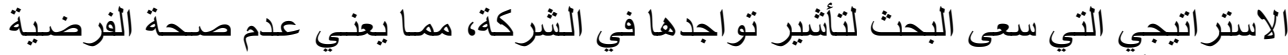

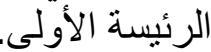

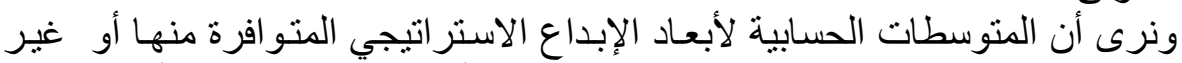

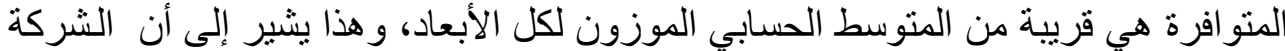

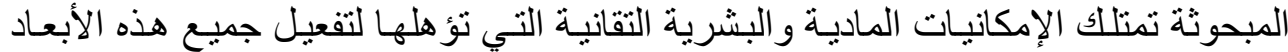

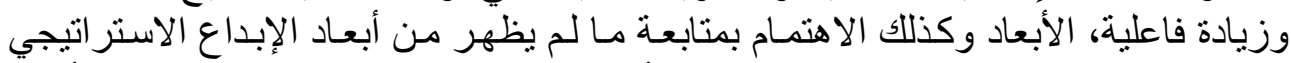

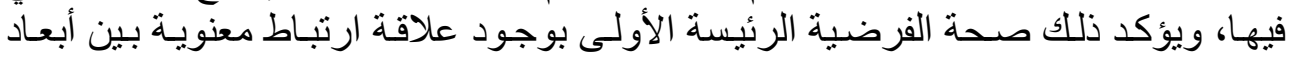

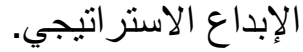

\begin{tabular}{|c|c|c|c|c|}
\hline \multicolumn{5}{|c|}{ المتوسط الحسابي لكل بعد ومقارنته مع المتوسط الحسابي الموزون } \\
\hline غير متوافر & متوافر & المتوسط الحسابي & المتوسط الحسابي & الأبعاد \\
\hline & & $r . \varepsilon 1 \leqslant \wedge$ & r.07r. & عملية الإبداع المدارة \\
\hline & & $r . \varepsilon 1 \leqslant \wedge$ & r.00ro & استشر اف الصناعة \\
\hline & & $r . \leqslant 1 \leqslant \wedge$ & $r . r \leq r_{0}$ & التو افق الاستر اتيجي \\
\hline & & $r . \leqslant 1 \leqslant \wedge$ & r.orIV & التقانات و القدر ات \\
\hline & & $r . \varepsilon 1 \leqslant \wedge$ & $r . \leq 7) V$ & تصور ات الزبون \\
\hline & & $r . \leqslant 1 \leqslant \wedge$ & $r .1 r \leqslant 0$ & الاستعدادية المنظمية \\
\hline$\longleftarrow$ & & $r . \varepsilon 1 \leqslant \wedge$ & $r . \varepsilon .1 T$ & التنفيذ المنظم \\
\hline
\end{tabular}


بهدف اختبار الفرضية الثانية التي تتص على وجود علاقة ارتباط التباط معنويـة بين أبعاد

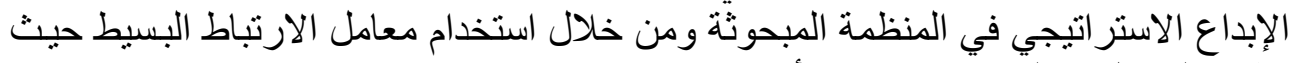
يظهر الجدول ه العلاقة بين هذه الأبعاد وكالاتي:

\section{- الجدول}

علاقة الارتباط بين أبعاد الإبداع الاستراتيجي

\begin{tabular}{|c|c|c|c|c|c|c|c|}
\hline & Y1 & Y2 & Y3 & Y4 & Y5 & Y6 & Y7 \\
\hline Y1 & 1 & & & & & & \\
\hline Y2 & $V \Psi \cdot * \star$ & 1 & & & & & \\
\hline Y3 & $7 \times 0^{* * *}$ & $V \leqslant \Gamma^{* * *}$ & 1 & & & & \\
\hline Y4 & フミ1** & イTト** & $(\vee)^{* *}$ & 1 & & & \\
\hline Y5 & $\prod イ$ & $V(Y)^{* *}$ & 791 ** & $\vee \wedge \Lambda^{* *}$ & 1 & & \\
\hline Y6 & $77 \lambda^{* *}$ & $V \cdot 0^{* *}$ & VYO** & $V \mu r^{* *}$ & V07** & $T$ & \\
\hline Y7 & $79 r^{* *}$ & $T V Y^{* *}$ & $797 * *$ & $V T_{T * *}$ & $T V Y^{* *}$ & Voq** & $T$ \\
\hline
\end{tabular}

** Correlation is significant at the 0.01 level

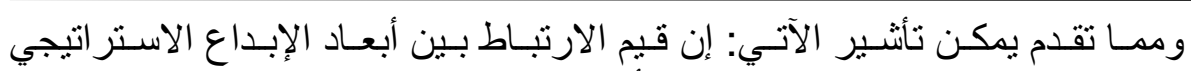

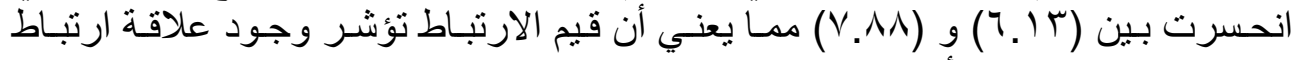

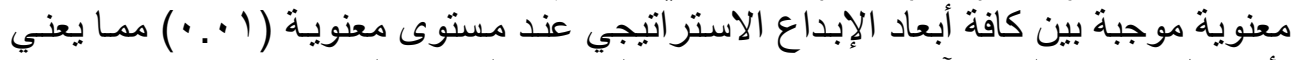

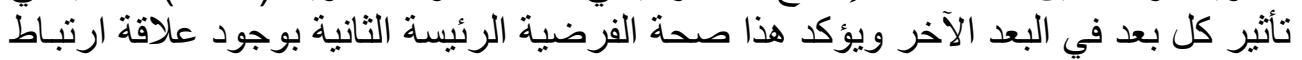
معنوية بين أبعاد الإبداع الاستر اتيجي في الإنظمة المذا المبحوثة.

الاستتتاجات والمقترحات

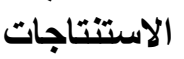

1 ـ تتمتع الثركة العامة لصناعة الأدوية و المستلزمات الطبية في نينوى بقدرة على إدارة

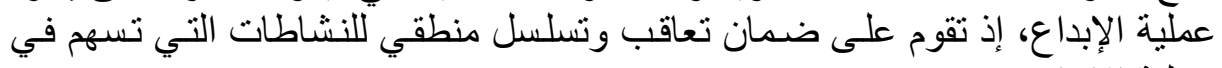
عملية الإبداع.

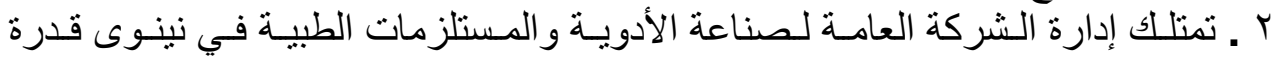

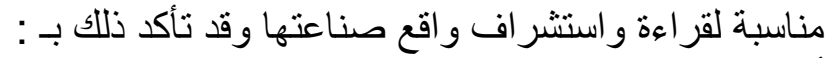

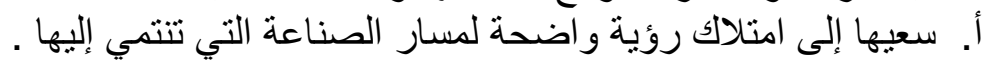

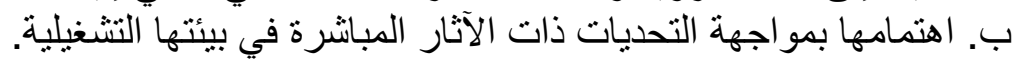

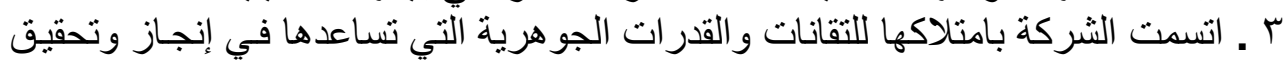

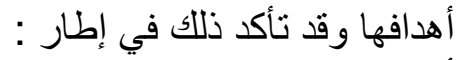

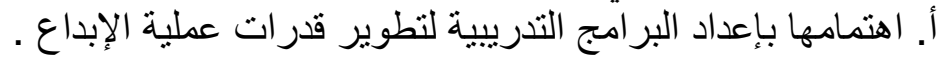

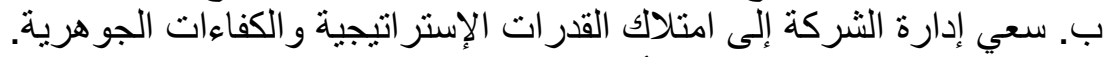

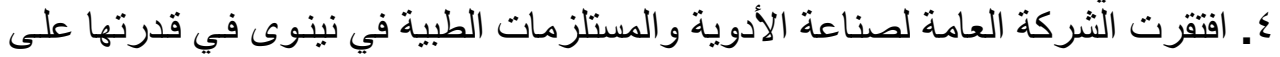

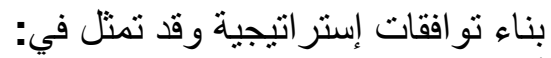
أ. ضعف قدرة الثركة على تحقيق حالة الدمج بين الأفكار الإبداعية المتأتية من داخل

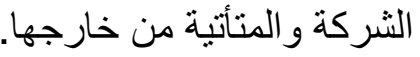

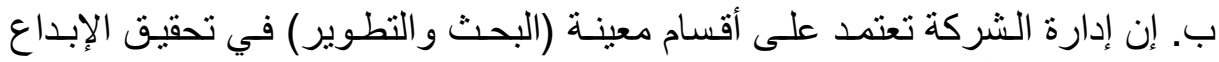
وليس من خلال مشاركة كافة الوحدات الوظئية ألئية في الثركة. 


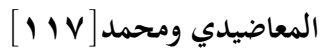

0. اتسمت الاستعدادية المنظمية في الثركة العامة لصناعة الأدوية و المستلزمات الطبية في

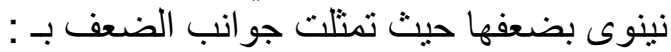

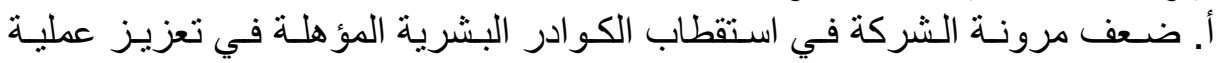

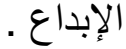
ب. ضعف و وداع ملاعمدة التغييرات الهيكلية في تحقيق الإبداع.

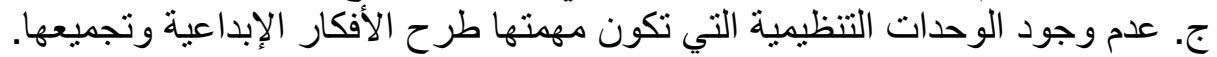

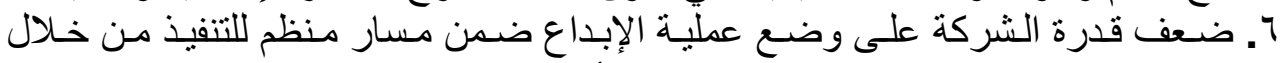
الامتلاك الضعيف للوسائل المناسبة لنقل الأفكار الإبداعية إلى المنفذين في الإدار ات

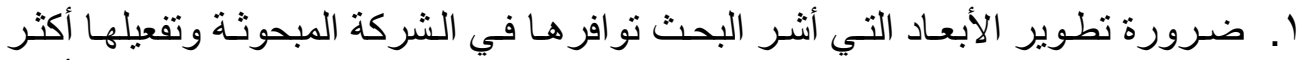

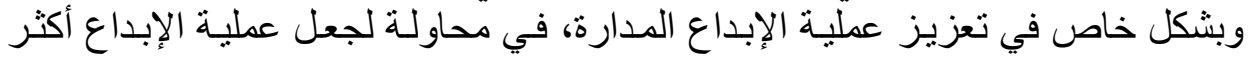

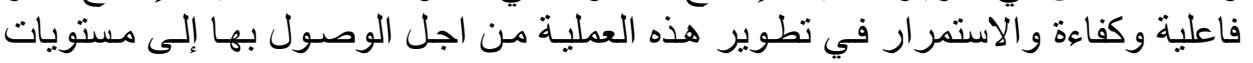

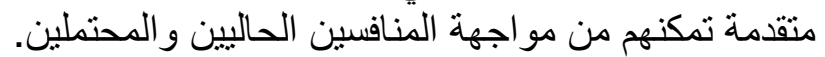

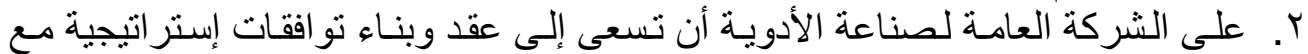

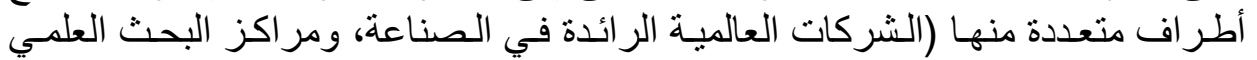

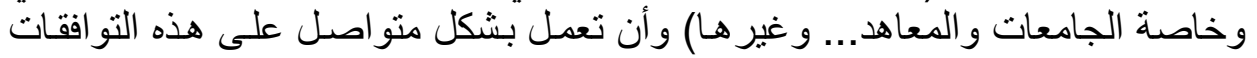

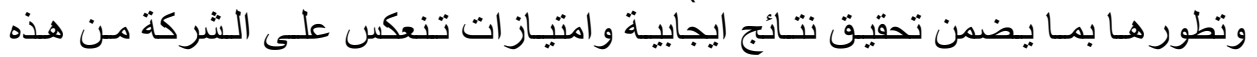

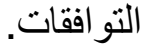

r. ضرورة قيام الشركة بتطوير قدر اتها في بناء هياكل تنظيمية مرنة قادرة على الاستجابة

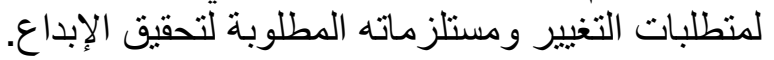

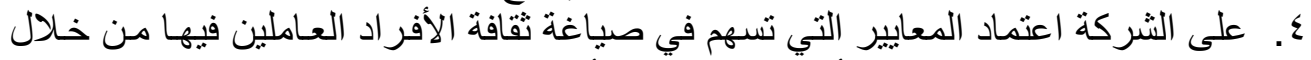

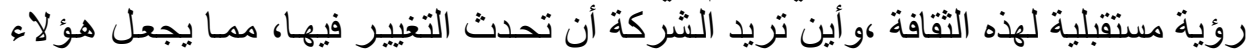

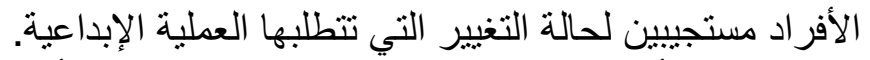

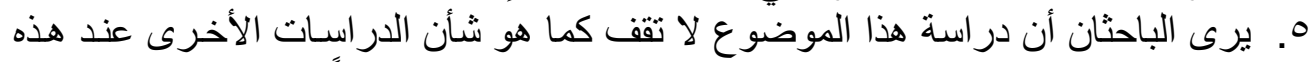

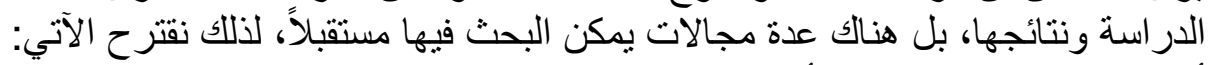

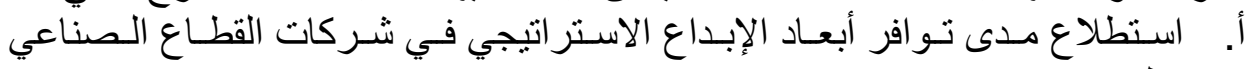

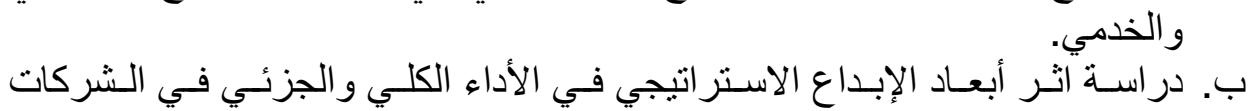
و المنظمات المختلفة.

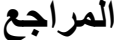

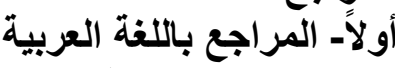

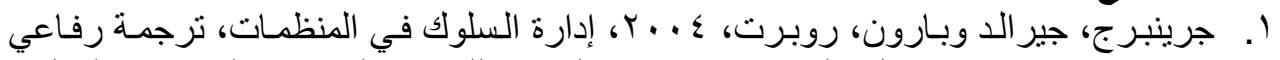

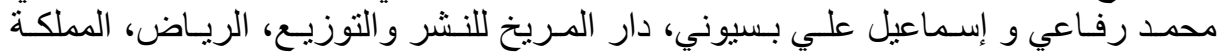
العربية السعودية.

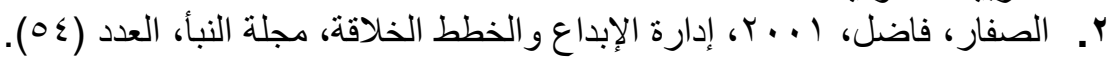




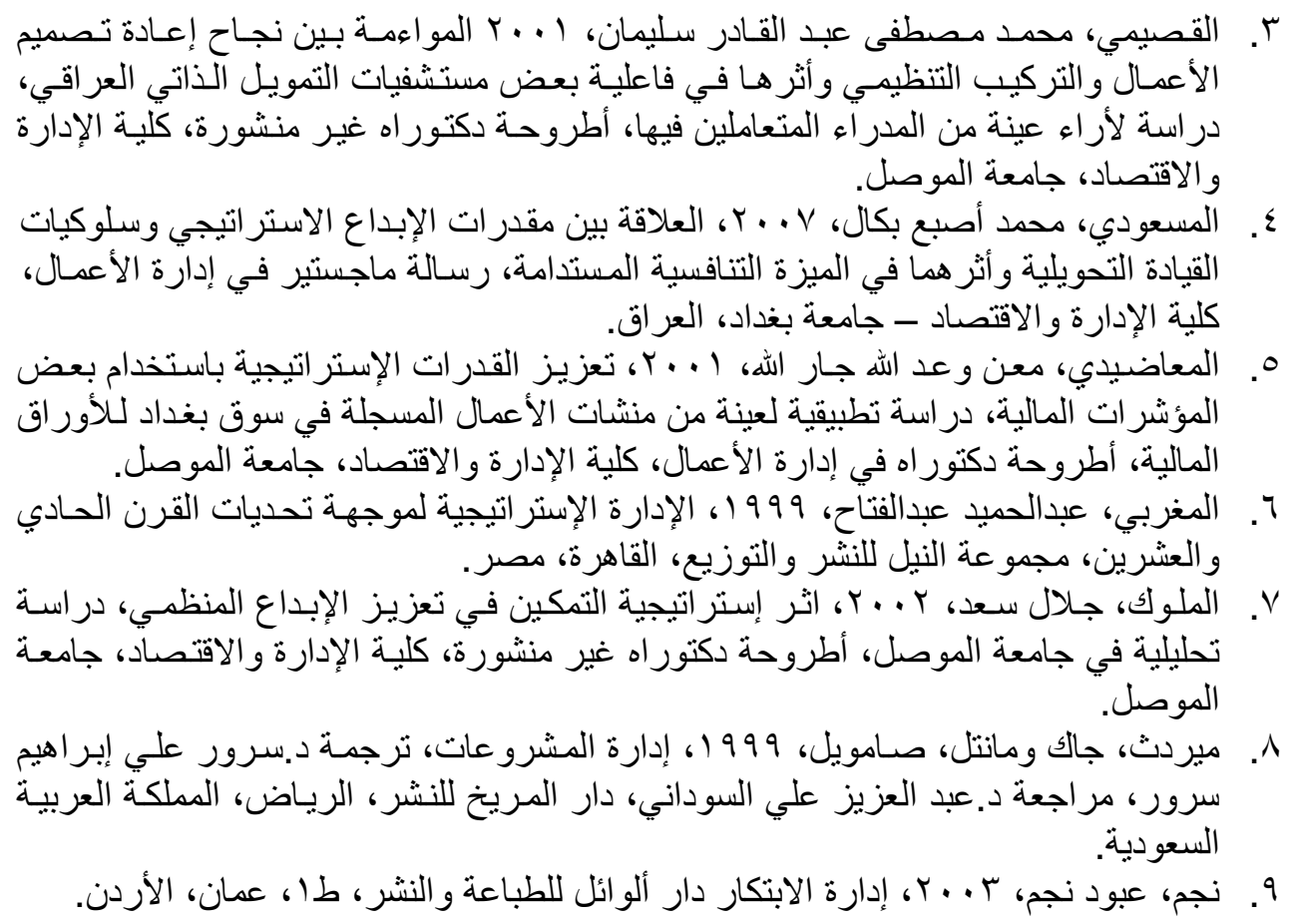

ثاتياً- المر اجع باللغة الأجنبية

1. Bechtold, B.L.,1997, Toward Participative Organizational culture evolution or evolution?, Empowerment in organization, VOL (5), No(1).

2. Berghman, Liselore, 2006, Strategic Innovation Capacity: A Mixed Method Study on Deliberate Strategic Learning Mechanisms, Ph. D Dissertation, University of Rotterdam.

3. Daft ,R.L. ,2001 organization : Theory and Design, south western collage publishing, Ohio, USA .

4. Derrick palmer and Soren Kaplan, 2007,"strategic innovation Blending strategy and Creativity to discover future business opportunities, www.innovation-point.com.

5. Govindarajan, V. and C. Trimble, 2005, Organizational DNA for Strategic Innovation. California Management Review, 47(3) spring.

6. Hamel ,G and Parhalad .C .K ,1999, Competing for the Future ,H.B.R ,JULY.August.

7. Jaffe D., 2001, organizational theory: Tension and changes McGraw-Hill Inter national edition Singapore, Thailand .

8. Leifer, Richard, 2000, Radical Innovation Research Technology Management, VOL (43) issue (6).

9. Lund, D.B.,2003, Organizational Culture and jop satisfaction Journal of Business and Industrial Marketing, vol (18),No (3).

10. Marion Debruyne and Marie schooraerts, 2006 ,Strategic innovation As The Alternative, octobar, www.innovation-point.com.

11. Michael Moeller, 2007, Strategic Innovation: Building New Growth businesses, EPDA Annual Conference May 18th, Beratergruppe Neuwaldegg, Vienna.

12. Mils ,R.,(1980),Macro organizational Behavior: sant Monica Galif, Roger, D. smith, 2002, The Role of the chief Technology officer in strategic innovation, project, execution and mentoring 3361 Rouse Road suit 200 ,Orland, Florida, www.titan .com. 


\section{المعاضيدي ومحمد[19]}

13. Roger L. Hammer,2002 , Strategic Innovation: The enging that propels Business, www. SBI Innovation.com .Toronto

14. Sniukas, Marc. 2007, Strategic Innovation a Synthesis of the Current Debate, www.Sniukas.com.

15. Soren Kaplan and Stu winby, 2007,Organizational Models for Innovation, Organizational Design that Support strategic Innovation and Growth . www. Innovation-point.com .

16. Willam mcelog: Implementing strategic change through project,International journa of project management, VOL (14), No (6). 1996.

17. www.innovation-point.com •

18. Yamaguchi I. ,2001, perceived organizational support for satisfying autonomy needs of Japanese white-collar work : A comparison between Japanese and U.S. -affiliated companies, journal of management psychology, VOL (16), NO (6). 